\title{
Estudo Comparativo dos Efeitos Hemodinâmicos e Ventilatórios da Ventilação Controlada a Volume ou a Pressão, em Cães Submetidos ao Pneumoperitônio *
}

\section{Hemodynamic and Ventilatory Effects of Volume or Pressure Controlled Ventilation in Dogs Submitted to Pneumoperitoneum. Comparative Study}

Antonio Roberto Carraretto, TSA ${ }^{1}$; Pedro Thadeu Galvão Vianna, TSA ${ }^{2}$; Armando Vieira de Almeida, TSA ${ }^{1}$; Eliana Marisa Ganem, TSA ${ }^{3}$

\begin{abstract}
RESUMO
Carraretto AR, Vianna PTG, Almeida AV, Ganem EM - Estudo Comparativo dos Efeitos Hemodinâmicos e Ventilatórios da Ventilação Controlada a Volume ou a Pressão, em Cães Submetidos ao Pneumoperitônio
\end{abstract}

JUSTIFICATIVA E OBJETIVOS: A ventilação com pressão controlada (PCV) está disponivel em aparelhos de anestesia, mas não existem estudos sobre o seu uso, durante o pneumoperitônio como $\mathrm{CO}_{2}(P P C)$. O objetivo deste estudo foi avaliar a ventilação controlada a pressão bem como as alterações hemodinâmicas e ventilatórias durante o PPC, comparando-a com a ventilação controlada a volume (VCV) convencionalmente utilizada.

MÉTODO: Dezesseis cães anestesiados com tiopental sódico, citrato de fentanil e brometo de pancurônio, foram divididos aleatoriamente em dois grupos: VC - ventilação controlada a volume $(n=8)$ e $P C$ - ventilação controlada a pressão $(n=8)$ Os parâmetros hemodinâmicos e ventilatórios foram monitorizados e registrados em 4 momentos: $M 1$ (antes do PPC), M2 (30 minutos após $P P C=10 \mathrm{mmHg}$ ), M3 (30 minutos após $P P C=15 \mathrm{mmHg}$ ) e $M 4$ (30 minutos após a deflação do $P P C$ ).

RESULTADOS: Com a aplicação do PPC ocorreu um aumento do volume corrente no grupo PC, aumento das pressões inspiratórias (máxima e de platô), diminuição da complacência proporcional ao aumento do PPC, aumento da freqüência cardíaca, manutenção da pressão arterial média com valores maiores no grupo VC em todos os momentos, aumento da pressão de átrio direito com diminuição significativa após a deflação, diminuição do $\mathrm{pH}$ sangüíneo durante o PPC com menor variação no grupo $P C$, maior estabilidade da pressão

\footnotetext{
${ }^{*}$ Recebido do (Received from) Laboratório Experimental do CET/SBA do Departamento de Anestesiologia da Faculdade de Medicina de Botucatu (FMB - UNESP), para o Programa de Pós-Graduação em Anestesiologia, Mestrado

1. Pós-Graduando (Mestrado) do Programa de Pós-Graduação em Anestesiologia da FMB - UNESP

2. Professor Titular do CET/SBA do Departamento de Anestesiologia da FMB - UNESP

3. Professora Adjunta Livre-Docente do CET/SBA do Departamento de Anestesiologia da FMB - UNESP

Apresentado (Submitted) em 08 de março de 2005

Aceito (Accepted) para publicação em 08 de setembro de 2005

Endereço para correspondência (Correspondence to)

Dr. Antonio Roberto Carraretto

Rua Cel. Monjardim, 289/1501

29015-500 Vitória, ES

E-mail: roberto@carraretto.med.br
}

(C) Sociedade Brasileira de Anestesiologia, 2005 parcial do $\mathrm{CO}_{2}$ no sangue arterial no grupo $P C$, sem alterações da pressão parcial do $\mathrm{O}_{2}$ no sangue arterial.

CONCLUSÕES: Apesar das diferenças de alguns parâmetros hemodinâmicos e ventilatórios, entre os dois modos de controle da ventilação, nas condições estudadas foi possível a utilização da ventilação controlada a pressão para procedimentos com a aplicação do PPC. É fundamental observar o controle rigoroso da ventilação alveolar, ajustando a pressão inspiratória para manter eliminação adequada do $\mathrm{CO}_{2}$ e garantir oxigenação.

Unitermos: ANIMAL, Cão; CIRURGIA, Abdominal: videolaparoscópica; VENTILAÇÃO: controlada mecânica

\section{SUMMARY}

Carraretto AR, Vianna PTG, Almeida AV, Ganem EM - Hemodynamic and Ventilatory Effects of Volume or Pressure Controlled Ventilation in Dogs Submitted to Pneumoperitoneum. Comparative Study

BACKGROUND AND OBJECTIVES: Pressure controlled ventilation (PCV) is available in anesthesia machines, but there are no studies on its use during $\mathrm{CO}_{2}$ pneumoperitoneum (CPP). This study aimed at evaluating pressure-controlled ventilation and hemodynamic and ventilatory changes during CPP, as compared to conventional volume controlled ventilation (VCV). METHODS: This study involved 16 dogs anesthetized with thiopental, fentanyl and pancuronium, which were randomly assigned to two groups: VC - volume controlled ventilation $(n=8)$ and $P C$ - pressure controlled ventilation $(n=8)$. Hemodynamic and ventilatory parameters were monitored and recorded in 4 moments: $M 1$ (before CPP), M2 (30 minutes after CPP $=10$ $\mathrm{mmHg}$ ), M3 (30 minutes after $C P P=15 \mathrm{mmHg}$ ) and $\mathrm{M} 4$ (30 minutes after deflation).

RESULTS: With CPP, there has been significant increase in tidal volume in PC group; there has been increase in airway pressures (peak and plateau), decrease in compliance with increase in CPP pressure, increase in heart rate, maintenance of mean blood pressure with higher values in the VC group in all stages; there was also increase in right atrium pressure with significant decrease after deflation, decrease in arterial $\mathrm{pH}$ with minor variations in $\mathrm{PC}$ group, greater arterial $p \mathrm{CO}_{2}$ stability in $P C$ group, and no significant changes in arterial $\mathrm{pO}_{2}$.

CONCLUSIONS: There were some differences in hemodynamic and ventilatory data between both ventilation control modes (VC and PC). It is possible to use pressure controlled ventilation during CPP, but the anesthesiologist must monitor and take a close look at alveolar ventilation, adjusting inspiratory pressure to ensure proper $\mathrm{CO}_{2}$ elimination and oxygenation.

Key Words: ANIMAL, Dog; SURGERY, Abdominal: videolaparoscopic; VENTILATION: mechanically controlled 


\section{INTRODUÇÃO}

O s procedimentos videolaparoscópicos apresentam benefícios para o paciente e para o sistema de saúde, estando o seu uso em crescimento em número e complexidade. Para a visualização do conteúdo e a operação do instrumental na cavidade abdominal é necessária a sua expansão. A técnica mais comum é a insuflação do dióxido de carbono $\left(\mathrm{CO}_{2}\right)$ na cavidade, para a criação de um pneumoperitônio. Apesar do $\mathrm{CO}_{2}$ ser um gás existente no sistema metabólico, a absorção e o aumento da concentração sangüínea, acima de determinados valores, produz efeitos cardiovasculares como o aumento do débito cardíaco 1,2 , o aumento da pressão arterial média, o aumento das concentrações da adrenalina e da noradrenalina plasmáticas ${ }^{1}$. O seu efeito vasodilatador pode levar a diminuição da resistência vascular sistêmica (SVR), quando não compensado pela ativação do sistema venoso de capacitância, através do sistema nervoso simpático ${ }^{1,2}$. Os relatos sobre os efeitos da inalação do $\mathrm{CO}_{2}$ na mecânica ventilatória de indivíduos não anestesiados, respirando espontaneamente, são controversos. A resistência das vias aéreas pode estar aumentada ${ }^{3}$, diminuída ${ }^{4}$ ou inalterada ${ }^{5}$.

O aumento da pressão intra-abdominal produz efeitos mecânicos sobre o abdômen e o tórax com alterações cardiovasculares e ventilatórias, desvia o diafragma no sentido cefálico, aumenta a pressão intratorácica, a expansão dos pulmões fica restringida pelo desvio da parte abdominal da parede torácica, a complacência pulmonar dinâmica diminui aproximadamente $50 \%$, aumentam as pressões inspiratórias de pico (Paw peek) e de platô (Paw plat) ${ }^{6-8}$.

Durante a anestesia geral ocorrem alterações da ventilação que necessitam do uso de ventilação controlada. A maioria dos ventiladores existentes nos aparelhos de anestesia disponibiliza a ventilação controlada a volume (VCV) e a ventilação limitada à pressão (PLV). Os novos equipamentos importados e alguns nacionais começam a incorporar a ventilação controlada a pressão (PCV), já existente e usual nos equipamentos das Unidades de Terapia Intensiva (UTI). Na VCV os parâmetros ajustados são o volume e o fluxo inspiratório, tanto o pico de fluxo quanto a sua forma. A freqüência ventilatória e a relação entre os tempos inspiratório e expiratório (Relação I:E) são dependentes destes ajustes. A pressão das vias aéreas será dependente da resistência e da complacência do sistema. Na PLV os parâmetros ajustados são a pressão limite e o fluxo inspiratório. O volume será dependente da resistência e da complacência. Dependendo do mecanismo de ciclagem, a relação I:E e a freqüência respiratória podem sofrer alterações. Na PCV os parâmetros a serem ajustados são: a pressão nas vias aéreas, o tempo inspiratório ou a relação I:E e a freqüência ventilatória. Estes parâmetros influenciarão sobre as características do fluxo inspiratório e o volume corrente, que deve ser monitorizada para uma ventilação adequada. Os ventiladores modernos utilizados nas UTI apresentam a modalidade PCV e o seu uso tem sido estudado em pacientes portadores de síndrome da angústia respiratória aguda (SARA). Por vezes estes pacientes são transferidos da UTI para o centro cirúrgico e existe a solicitação para que continuem na mesma modalidade de ventilação. Nas modalidades de ventilações descritas, é possível o acréscimo de pressão positiva ao final da expiração(PEEP), com a finalidade de evitar ou diminuir o colapso e melhorar o recrutamento alveolar. Alterações hemodinâmicas ${ }^{9,10}$ e renais foram avaliadas em outros estudos, com métodos semelhantes. O objetivo deste estudo foi avaliar a aplicabilidade da ventilação controlada a pressão (PCV) em anestesia, com a presença do pneumoperitônio com $\mathrm{CO}_{2}$, através da medida dos parâmetros hemodinâmicos e respiratórios, bem como compará-la com a ventilação controlada a volume (VCV) convencionalmente utilizada.

\section{MÉTODO}

Após aprovação pelo Comitê de Ética em Pesquisa Animal da Faculdade de Medicina de Botucatu - UNESP, foram utilizados 16 cães adultos, de ambos os sexos, com peso entre 15 e $23 \mathrm{~kg}$, sem raça definida, fornecidos pelo Biotério do Campus de Botucatu da UNESP, deixados em jejum de alimentos sólidos por 12 horas e livres para beber água. Os animais foram aleatoriamente divididos, por sorteio, em dois grupos de oito animais cada: Grupo VC - Ventilação controlada a volume (VCV) e o Grupo PC - Ventilação controlada a pressão (PCV).

Seqüência experimental: 1) Verificação, teste e ajuste dos equipamentos (aparelho de anestesia, monitorização, bombas de infusão, aquecedor de soluções, aquecedor do animal, insuflador de gás); 2) Registro do peso, comprimento e sexo do animal; 3) Indução da anestesia com a administração, por via venosa, de tiopental sódico $\left(15 \mathrm{mg} \cdot \mathrm{kg}^{-1}\right)$, citrato de fentanil $\left(0,015 \mathrm{mg} \cdot \mathrm{kg}^{-1}\right)$ e brometo de pancurônio $(0,07$ mg. $\mathrm{kg}^{-1}$ ); 4) Intubação orotraqueal (IOT), com tubo com balão (calibre 8,5 mm ID) e verificação; 5) Conexão do adaptador do Biomonitor Datex $A S 3^{\circledR}$, para medida da ventilação e análise dos gases inspirados e expirados, ao conector em $Y$ do filtro do absorvedor de $\mathrm{CO}_{2}$ do aparelho de anestesia Ohmeda Excel $210 \mathrm{SE}^{\circledR}$, com fluxo de gases frescos $(\mathrm{FGF})=$ $0,5 \mathrm{~L}$. $\mathrm{min}^{-1}$ de oxigênio a $\left.100 \%\left(\mathrm{~F}_{\mid} \mathrm{O}_{2}=1,0\right) ; 6\right)$ Início da ventilação controlada mecânica com o ventilador Ohmeda $7900^{\circledR}$, acoplado ao aparelho de anestesia, na modalidade selecionada, VCV ou PCV, determinada pelo grupo e ajuste dos parâmetros para manter a estabilidade ventilatória desejada; 7) Instalação da monitorização não-invasiva: ECG contínuo na derivação $D_{\| l}$, oxímetro de pulso e sensor do termômetro esofágico; 8) Dissecção e cateterismo da veia femoral direita, para a infusão de líquidos e medicamentos e início da hidratação venosa com solução de Ringer, com volume de 6 $\mathrm{mL} . \mathrm{kg} \cdot \mathrm{h}^{-1}$, através de bomba de infusão e aquecedor com controle da temperatura a $36,5^{\circ} \mathrm{C} ; 9$ ) Dissecção e cateterismo da artéria femoral esquerda, para a monitorização da pressão arterial média (PAM) e coleta de sangue para as gasometrias, com conexão ao transdutor de medida do monitor Datex AS3 ${ }^{\circledR}$, previamente calibrado; 10) Dissecção da veia jugular direita com introdução do cateter de Swan-Ganz 7F até a artéria pulmonar, para as medidas hemodinâmicas; 11) 
Realização de minilaparotomia mediana, para a introdução de um cateter de polietileno calibre $14 \mathrm{~F}$ para a insuflação do gás e controle da PIA, com o insuflador Olympus Surgical Insufflator $15-\mathrm{L}^{\circledR}$ e retirada do gás no momento M3; 12) Cobertura do animal com manta para aquecimento, pela insuflação de ar aquecido $\left(42-46^{\circ} \mathrm{C}\right)$ por meio do equipamento WarmTouch ${ }^{\circledR}$.

A manutenção da anestesia foi realizada com tiopental (6 $\left.\mathrm{mg} \cdot \mathrm{kg}^{-1} \cdot \mathrm{h}^{-1}\right)$ e citrato de fentanil $\left(0,006 \mathrm{mg} \cdot \mathrm{kg}^{-1} \cdot \mathrm{h}^{-1}\right)$ em infusão contínua, com a bomba de infusão $A N N E^{\circledR}$. Doses adicionais de brometo de pancurônio $\left(0,02 \mathrm{mg} \cdot \mathrm{kg}^{-1}\right)$ e citrato de fentanil $\left(0,005 \mathrm{mg} \cdot \mathrm{kg}^{-1}\right)$ foram administradas, de acordo com a necessidade.

Ainsuflação do $\mathrm{CO}_{2}$ na cavidade abdominal para atingir uma PIAde $10 \mathrm{mmHg}$ (após M1) e $15 \mathrm{mmHg}$ (após M2) e a retirada do gás para uma PIA de $0 \mathrm{mmHg}$ (após M3).

Após o término do experimento, a eutanásia do animal foi realizada com injeção venosa de $10 \mathrm{~mL}$ de cloreto de potássio a $19,1 \%$.

Foram estudados os seguintes parâmetros: peso (kg), comprimento $(\mathrm{cm})$, superfície corporal (calculada $-\mathrm{m}^{2}$ ), sexo $(\mathrm{M} / \mathrm{F})$, freqüência respiratória $\left(\mathrm{f}\right.$ - $\left.\mathrm{mov} \cdot \mathrm{min}^{-1}\right)$, volume corrente $\left(\mathrm{V}_{\mathrm{T}}-\mathrm{mL}\right)$, pressão inspiratória (de pico) máxima (Paw peek - $\mathrm{cmH}_{2} \mathrm{O}$ ), pressão inspiratória de platô (Paw plat - $\mathrm{cmH}_{2} \mathrm{O}$ ), complacência torácica $\left(\mathrm{C}_{\mathrm{T}}-\mathrm{mL} . \mathrm{cm}^{-1} \mathrm{H}_{2} \mathrm{O}\right)$, pressão parcial do $\mathrm{CO}_{2}$ no final da expiração $\left(\mathrm{P}_{\mathrm{ET}} \mathrm{CO}_{2}-\mathrm{mmHg}\right)$, freqüência cardíaca ( $F C$ - bat. $\min ^{-1}$ ), pressão arterial média (PAM $\mathrm{mmHg}$ ), pressão média do átrio direito (RAP - $\mathrm{mmHg}$ ), $\mathrm{pH}$ arterial $(\mathrm{pH})$, pressão parcial do dióxido de carbono no sangue arterial $\left(\mathrm{PaCO}_{2}-\mathrm{mmHg}\right)$, pressão parcial do oxigênio no sangue arterial $\left(\mathrm{PaO}_{2}-\mathrm{mmHg}\right)$.

Após a instalação do cateter para a realização do PPC e da monitorização procedia-se à contagem de 30 minutos, buscando-se a estabilidade ventilatória, através do ajuste do volume corrente (VCV) ou da pressão inspiratória (PCV), para a manutenção da $\mathrm{P}_{\mathrm{ET}} \mathrm{CO}_{2}$ dentro dos limites desejados (35 a 45 $\mathrm{mmHg}$ ), com a freqüência respiratória regulada para 10 ipm. min $^{-1}$, relação inspiração-expiração de 1:2 e uma PEEP de $3 \mathrm{cmH}_{2} \mathrm{O}$. As medidas ventilatórias foram realizadas com o monitor Datex $A S 3^{\circledR}$, com o conector do sensor junto ao conector do tubo traqueal. Este procedimento já foi validado anteriormente ${ }^{11}$.

As medidas ventilatórias, hemodinâmicas, sangüíneas e de temperatura foram realizadas nos momentos $M 1, M 2, M 3$ e M4, sendo: M1-imediatamente após o término do período de estabilização de 30 minutos com uma PIA $=0 \mathrm{mmHg}, \mathrm{M} 2-30$ minutos após o PPC, para uma PIA $=10 \mathrm{mmHg}$, M3- 30 minutos após o PPC, para uma PIA $=15 \mathrm{mmHg}, \mathrm{M} 4-30$ minutos após o PPC, para uma PIA $=0 \mathrm{mmHg}$.

A Análise de Variância inteiramente aleatorizada foi utilizada para as variáveis do peso, do comprimento e da área de superfície corpórea, para a comparação dos dois grupos $^{12}$.

A Análise de Perfil foi utilizada para as demais variáveis, avaliadas nos quatro momentos ${ }^{13}$.

\section{RESULTADOS}

As medidas de peso, comprimento e área de superfície corpórea dos animais não apresentaram diferenças significativas entre os dois grupos e estão relacionados na tabela I.

Tabela I - Valores Antropométricos dos Animais, nos Grupos Estudados (Média $\pm \mathrm{DP}$ )

\begin{tabular}{lccc}
\hline Grupos & Peso $(\mathrm{kg})$ & Comprimento $(\mathrm{cm})$ & ASC $\left(\mathrm{m}^{2}\right)$ \\
\hline VC $(n=8)$ & $20,3 \pm 3,1$ & $105,1 \pm 8,0$ & $0,8 \pm 0,1$ \\
PC $(n=8)$ & $18,6 \pm 1,5$ & $100,3 \pm 4,1$ & $0,7 \pm 0,0$ \\
\hline
\end{tabular}

Os valores do volume corrente, regulados para manter a $\mathrm{P}_{\mathrm{ET}} \mathrm{CO}_{2}$ entre 35 e $45 \mathrm{mmHg}$, não apresentaram diferenças significativas entre os grupos VC e PC em cada momento. No grupo VC os valores mantiveram-se constantes e no grupo $\mathrm{PC}$ ocorreu um aumento após o pneumoperitônio, que não retornou aos valores iniciais (Figura 1).

A pressão inspiratória máxima aumentou com o aumento da PIA nos dois grupos, com tendência a valores maiores no grupo VC em M1 e M4, com retorno aos valores iniciais após a deflação (Figura 2).

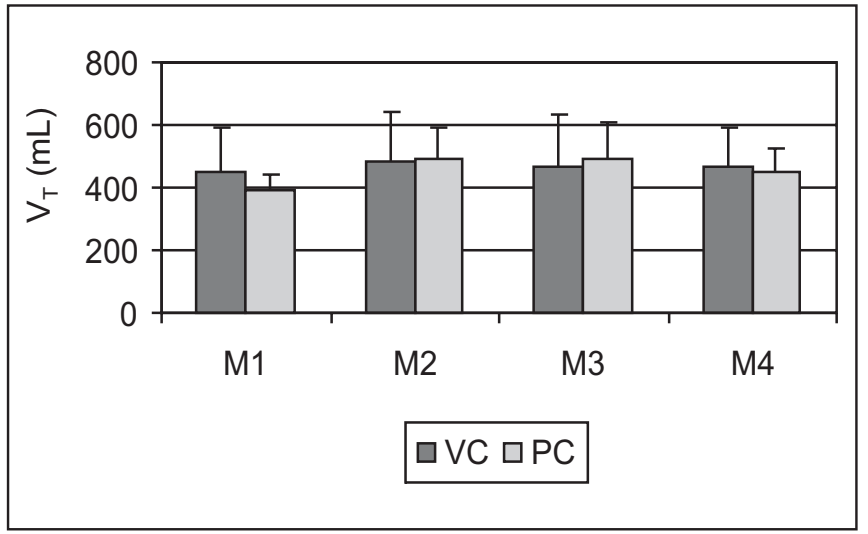

Figura 1 - Volume Corrente $(\mathrm{mL})$ Relativos aos Grupos e Momentos Estudados (Média \pm DP)

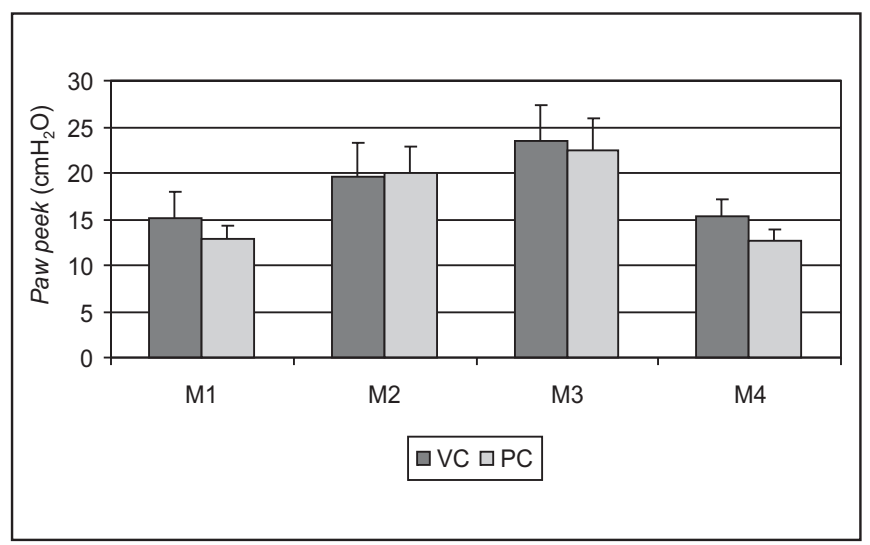

Figura 2 - Pressão Inspiratória Máxima $\left(\mathrm{cmH}_{2} \mathrm{O}\right)$ Relativos aos Grupos e Momentos Estudados (Média \pm DP) 
A pressão inspiratória de platô aumentou com o aumento da PIAe retornou ao valor inicial após a deflação, apresentando o mesmo comportamento nos dois grupos (Figura 3).

Os valores da complacência diminuíram com o aumento da PIA nos dois grupos. Após a deflação ocorreu o retorno aos valores iniciais no grupo VC e valores maiores que os iniciais no grupo PC (Figura 4).

Os valores da pressão parcial do dióxido de carbono no final da expiração do grupo VC apresentaram uma tendência a serem maiores do que os do grupo PC, durante o pneumoperitônio, com retorno aos valores iniciais após a deflação. O grupo PC apresentou maior uniformidade dos valores (Figura 5).

Os valores da freqüência cardíaca dos grupos VC e PC apresentaram o mesmo comportamento, com os seus maiores valores em M2 e M3 (Figura 6).

Os valores da pressão arterial média do grupo VC foram maiores do que os do grupo PC. Não houve diferença entre os momentos nos dois grupos (Figura 7).

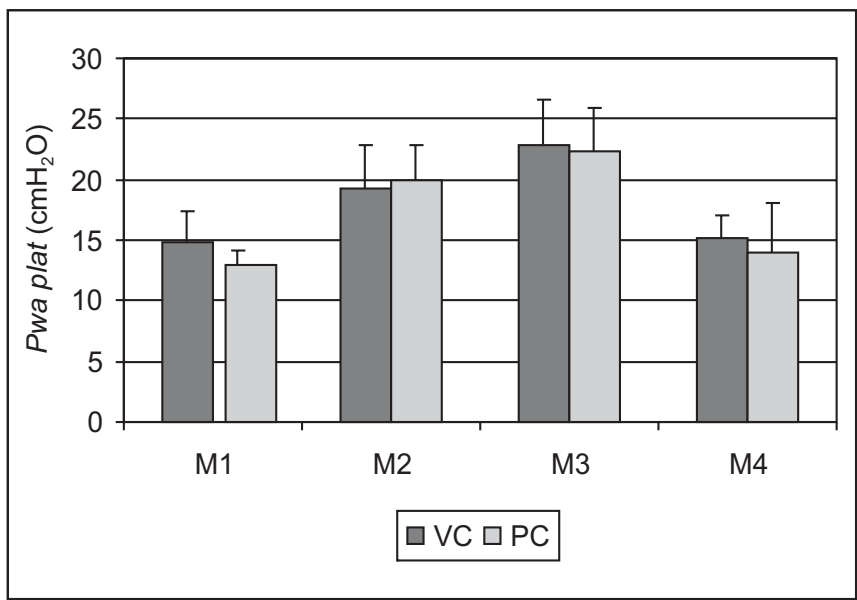

Figura 3 - Pressão Inspiratória de Platô $\left(\mathrm{cmH}_{2} \mathrm{O}\right)$ Relativos aos Grupos e Momentos Estudados (Média $\pm \mathrm{DP}$ )

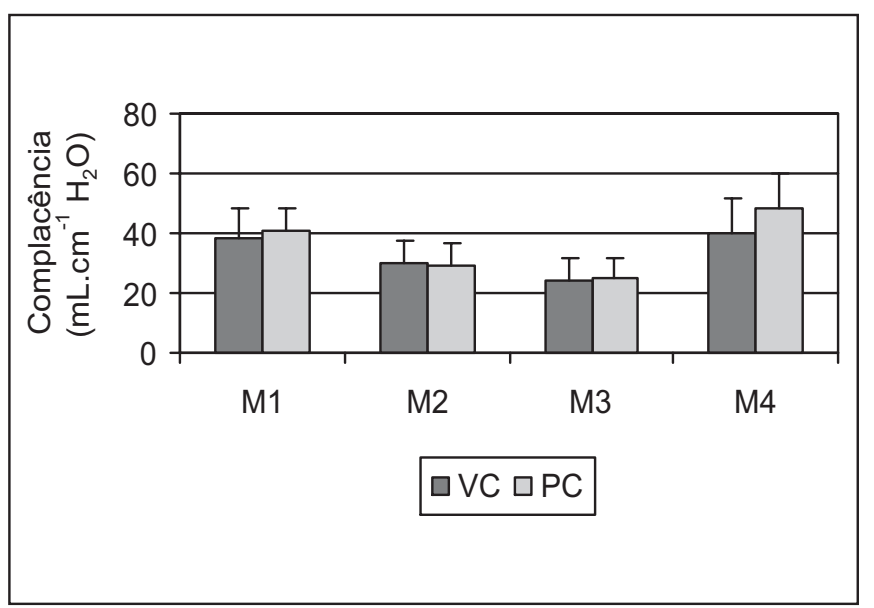

Figura 4 - Complacência $\left(\mathrm{mL} . \mathrm{cm}^{-1} \mathrm{H}_{2} \mathrm{O}\right)$ Relativos aos Grupos e Momentos Estudados (Média \pm DP)

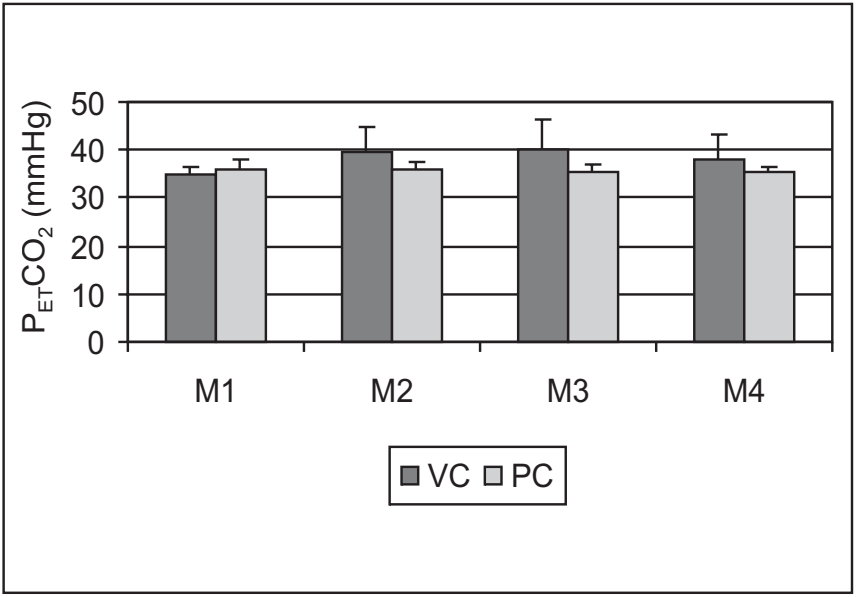

Figura 5 - Pressão Parcial do $\mathrm{CO}_{2}$ Relativos aos Grupos e Momentos Estudados (Média \pm DP)

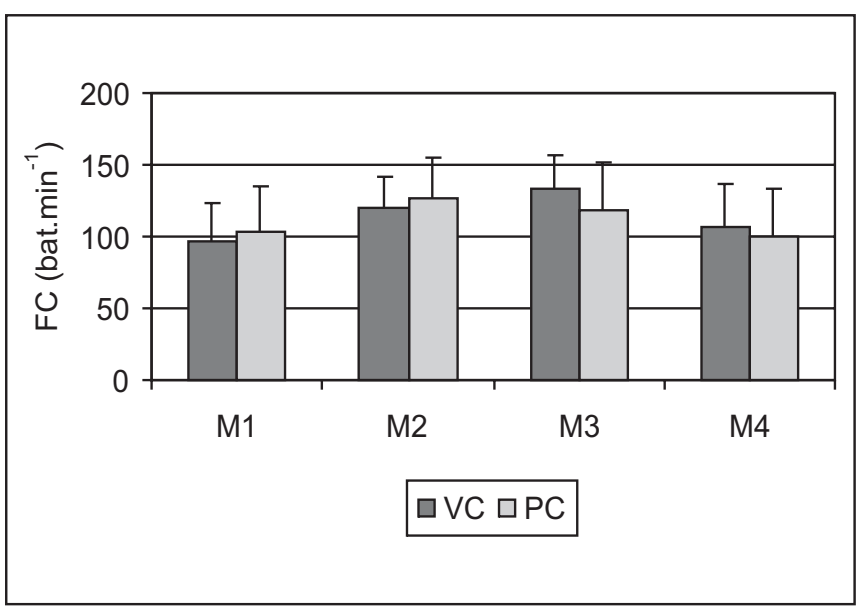

Figura 6 - Freqüência Cardíaca (bat. $\min ^{-1}$ ) Relativos aos Grupos e Momentos Estudados (Média \pm DP)

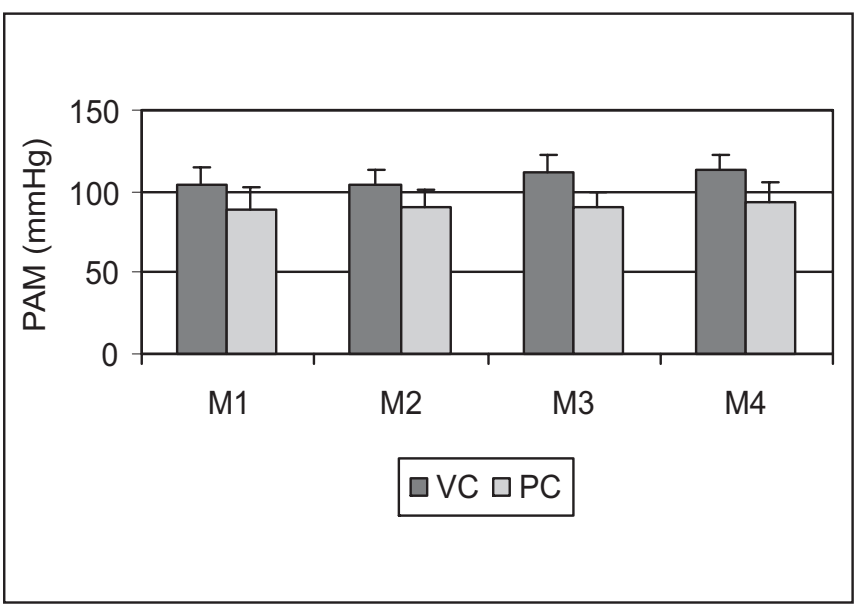

Figura 7 - Pressão Arterial Média $(\mathrm{mmHg})$ Relativos aos Grupos e Momentos Estudados (Média $\pm \mathrm{DP}$ )

Revista Brasileira de Anestesiologia Vol. 55, № 6, Novembro - Dezembro, 2005 
Os valores da pressão do átrio direito aumentaram durante o pneumoperitônio, diminuíram após a deflação, com diminuição significativa no grupo PC (Figura 8).

Os valores do $\mathrm{pH}$ sangüíneo foram diferentes entre os momentos. No grupo VC eles diminuíram durante o pneumoperitônio. No grupo PC apresentaram uma menor variação (Figura 9).

Os valores da pressão parcial de dióxido de carbono no sangue arterial apresentaram diferenças entre os grupos, com aumento (M2) e tendência a aumento (M3) no grupo VC, durante o pneumoperitônio, e estabilidade no grupo PC (Figura 10).

Os valores da pressão parcial de oxigênio no sangue arterial apresentaram o mesmo comportamento, sem diferenças entre grupos e momentos (Figura 11).

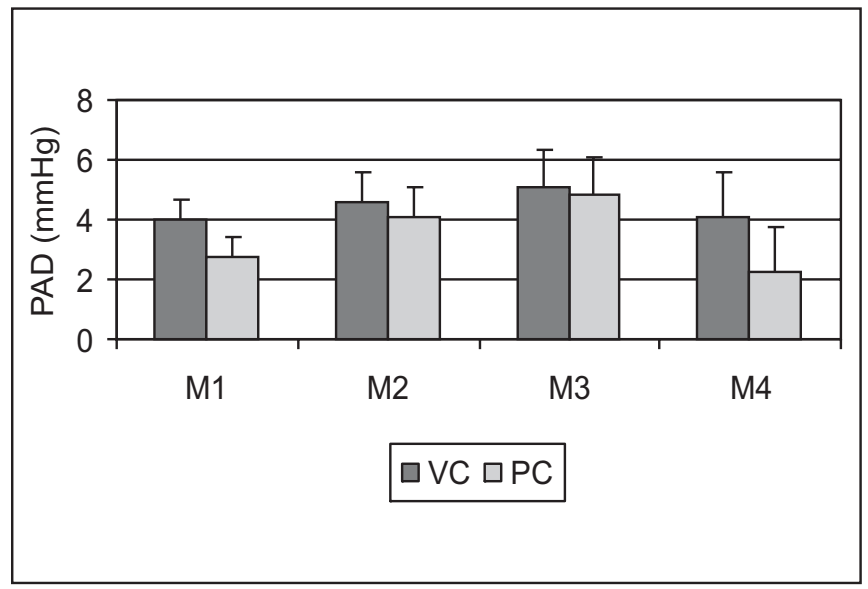

Figura 8 - Pressão Média do Átrio Direito $(\mathrm{mmHg})$ Relativos aos Grupos e Momentos Estudados (Média \pm DP)

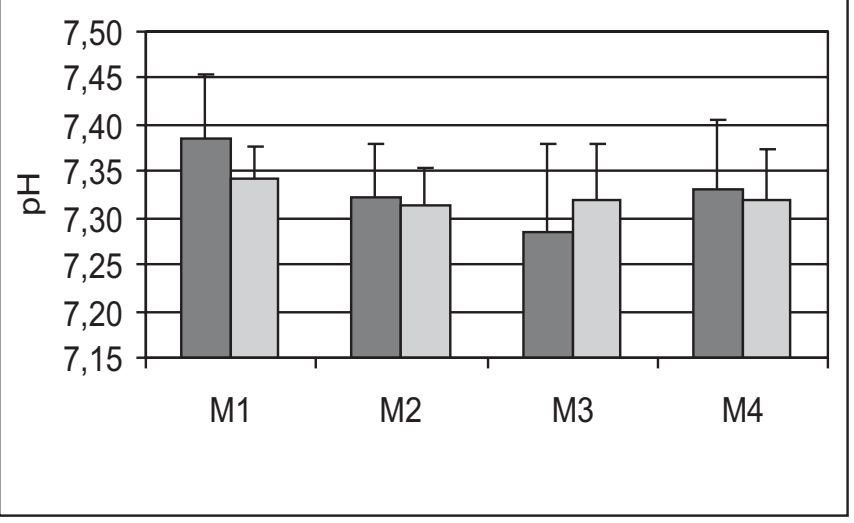

Figura 9 - pH Arterial Relativos aos Grupos e Momentos Estudados (Média \pm DP)

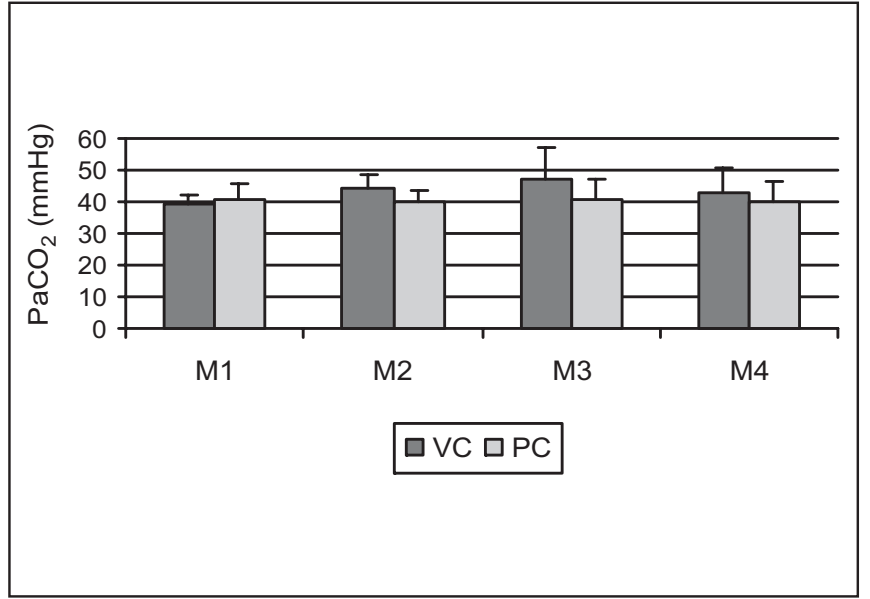

Figura 10 - Pressão Parcial de Dióxido de Carbono no Sangue Arterial $(\mathrm{mmHg})$ Relativos aos Grupos e Momentos Estudados (Média \pm DP)

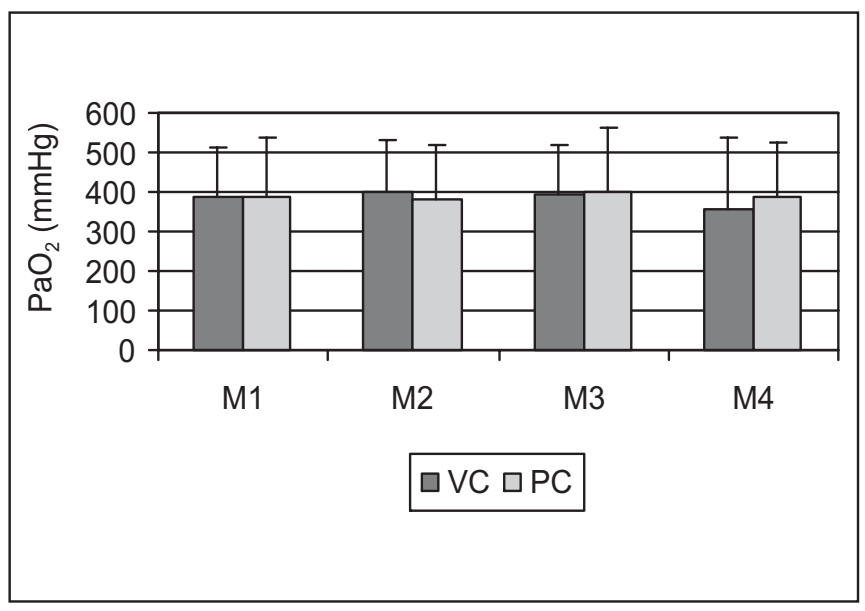

Figura 11 - Pressão Parcial de Oxigênio no Sangue Arterial $(\mathrm{mmHg})$ Relativos aos Grupos e Momentos Estudados (Média \pm DP)

\section{DISCUSSÃo}

$\mathrm{Na}$ ventilação controlada mecânica, dentre os principais controles disponíveis, estão o volume e a pressão, objetivos do presente estudo. Estes controles podem ser pré-fixados individualmente ou conjugados, gerando a ventilação controlada a volume (VCV) ou a ventilação controlada a pressão (PCV). Combinações de controles como volume-alvo, pressão controlada e ciclagem a tempo estão entre as novas modalidades disponíveis, denominadas modos de controle duplo.

Neste estudo, para um melhor controle dos diversos parâmetros a serem estudados, a freqüência respiratória foi fixada em 10 mov. min $^{-1}$, semelhante a estudos já existentes ${ }^{7,14}$ e a relação inspiração-expiração em 1:2. Estes valores permitiram uma adequada ventilação dos animais, comprovada pelos valores obtidos nas análises da $\mathrm{P}_{\mathrm{ET}} \mathrm{CO}_{2}, \mathrm{PaCO}_{2}$ e $\mathrm{PaO}_{2}$. 
Houve a necessidade de aumento do volume corrente, por ajuste no controle de volume do grupo VC ou no controle de pressão do grupo $\mathrm{PC}$, para a manutenção da $\mathrm{P}_{\mathrm{ET}} \mathrm{CO}_{2}$ nos valores pretendidos. Outro estudo demonstra a necessidade de aumento do volume minuto respiratório, de $20 \%$ a $30 \%$, para a eliminação do $\mathrm{CO}_{2}$ absorvido, pelo PPC ${ }^{15}$.

$\mathrm{Na}$ VCV o volume a ser administrado é um fator fixo (pré-determinado) juntamente com a freqüência ventilatória e a relação I:E. O ventilador gera um fluxo controlado, que é interrompido ao final da inspiração, podendo após este existir uma pausa inspiratória (com a manutenção de uma pressão de platô) ou a ciclagem para a fase expiratória. A pressão será resultante da mecânica ventilatória, como as alterações sobre a resistência e a complacência. Com o aumento da PIA, o aumento da pressão intratorácica promove um aumento da pressão das vias aéreas. O volume ventilatório minuto sofre uma pequena ou nenhuma alteração e a ventilação alveolar, responsável pela retirada do $\mathrm{CO}_{2}$ alveolar, é mantida.

Na PCV, o ventilador inicia o ciclo com um fluxo de demanda necessário para manter uma pressão pré-determinada (fator fixo). Quando esta pressão é atingida o fluxo diminui gradativamente (fluxo desacelerado) o suficiente para manter a pressão. O volume corrente depende da pressão regulada, do tempo inspiratório e, como em todo o modo por pressão, será influenciado pelas variações na mecânica ventilatória (complacência e resistência) ${ }^{16}$.

Durante a PCV, na abertura da válvula inspiratória, o fluxo de gás é alto e grande parte do volume é distribuída logo no início da fase inspiratória. A válvula do mecanismo de controle da pressão regula o fluxo inspiratório, mantendo a pressão constante e permitindo melhor distribuição do gás para os alvéolos de maior constante de tempo, sem provocar hiperdistensão - por controlar a pressão dos alvéolos. Para aumentar a ventilação alveolar, é necessário o aumento da freqüência ventilatória ou da pressão inspiratória.

O aumento na resistência das vias aéreas ou a diminuição da complacência pulmonar reduzem o volume corrente e conseqüentemente a ventilação alveolar. Devido aos efeitos da PIA sobre a pressão intratorácica, para cada variação na PIA pode ser necessária uma correção dos parâmetros ventilatórios, geralmente uma nova pressão, para compensar este efeito de perda ou de ganho do volume corrente.

Em estudo comparativo entre a PCV e a VCV, em pacientes com síndrome da angústia respiratória aguda (SARA) grave, foi observada uma menor redução no débito cardíaco no grupo da PCV, relatada como o resultado de uma melhora da pós-carga do ventrículo direito, devido a um melhor recrutamento alveolar, a uma redução da resistência vascular pulmonar e a diminuição da pressão intratorácica que facilitou uma melhor pré-carga de ambos os ventrículos ${ }^{17}$.

Em outro estudo comparativo em pacientes submetidos à ventilação monopulmonar, foi observado que a Paw peek, a Paw plat e o shunt pulmonar foram significativamente maiores durante a VCV enquanto a $\mathrm{PaCO}_{2}$ foi maior durante a $P C V$ e concluíram que a PCV é uma alternativa à VCV, em pacientes que necessitem de ventilação monopulmonar, e pode ser superior à VCV em pacientes com doenças respiratórias ${ }^{18}$

No pós-operatório de pacientes de cirurgia cardíaca, os dois controles mostraram efeitos comparáveis em pacientes com função cardíaca preservada ou deprimida, mas nos pacientes com a PCV ocorreram maiores valores de índice cardíaco, diminuição da SVR e menores valores das pressões inspiratórias, quando comparados aos pacientes da VCV ${ }^{19}$. APCV tem sido empregada em UTI, para o tratamento de pacientes com doenças respiratórias graves, com melhores resultados sobre a oxigenação e melhor prevenção da lesão ao tecido pulmonar ${ }^{20}$. Estes pacientes, já com alterações cardiovasculares e/ou respiratórias, quando submetidos aos procedimentos que exijam o uso do PPC, apresentam maior dificuldade do controle da ventilação e da manutenção da normocapnia e da oxigenação. Existem situações com a necessidade de cirurgias e o uso do pneumoperitônio em pacientes que já estejam fazendo o uso da PCV, ou que esta modalidade seja a melhor indicação.

Uma das principais características da PCV é a dependência entre a mecânica respiratória do paciente, o fluxo e o volume inspiratório. A PCV previne a ocorrência de pressões elevadas, determinantes do mecanismo de lesão pulmonar, na presença de diferenças de resistência e complacência alveolares, por manter constante a pressão nas vias aéreas ${ }^{21}$. A absorção máxima ocorre com pressões relativamente baixas, de $10 \mathrm{mmHg}$. O gás absorvido é eliminado pelos pulmões, por vezes até no período pós-operatório, já que volumes de mais de 120 litros podem ser estocados em nosso organismo. Os principais reservatórios são os ossos ${ }^{22}$.

$\mathrm{Na}$ ventilação controlada a pressão ocorreu a necessidade de aumento do volume corrente após a aplicação do pneumoperitônio.

A pressão inspiratória máxima é o valor máximo da pressão gerada para insuflar os pulmões. Ela depende das propriedades elásticas e resistivas do sistema respiratório. Os componentes elásticos geram a pressão necessária para mudar o volume pulmonar enquanto os resistivos representam a pressão necessária para gerar o fluxo gasoso ${ }^{16}$.

Durante a aplicação do PPC ocorreu um aumento proporcional da Paw peek, sem diferença significativa entre os modos de controle VC e PC.

Aaplicação do PPC determinou um aumento proporcional da Paw plat, que retornou ao valor inicial após a retirada, sem diferença significativa entre a ventilação controlada a volume e a ventilação controlada a pressão.

A concentração do $\mathrm{CO}_{2}$ no final da expiração, medida pela capnometria, foi o alvo de controle do estudo. Para manter a $\mathrm{P}_{\mathrm{ET}} \mathrm{CO}_{2}$ dentro dos limites programados no grupo $\mathrm{VC}$ foi ajustado o volume e no grupo PC foi ajustada a pressão inspiratória.

Foi observado que durante a insuflação prolongada do $\mathrm{CO}_{2}$ na cavidade abdominal, a absorção pelo peritôneo leva a um aumento da sua concentração sangüínea e da sua eliminação. O padrão é caracterizado por uma fase inicial, com um rápido aumento na eliminação do $\mathrm{CO}_{2}\left(27-37 \mathrm{~mL} \cdot \mathrm{min}^{-1}\right) \log$ o após o início da insuflação, seguido de uma fase mais lenta, 
devido a distensão da superfície peritoneal com a compressão dos vasos peritoneais ${ }^{23}$. Este padrão de eliminação pulmonar bifásico também foi observado em modelo animal ${ }^{24}$.

$\mathrm{O} \mathrm{CO}_{2}$ apresenta boa solubilidade sangüínea gerando uma solução ácida. Para evitar a hipercapnia e a acidose respiratória, a ventilação deve ser aumentada em cerca de $20 \%$ a $30 \% 15,22$

Durante o PPC, a $\mathrm{P}_{\mathrm{ET}} \mathrm{CO}_{2}$ aumenta progressivamente com o tempo atingindo o seu valor máximo após 40 minutos, se a ventilação for mantida constante ${ }^{25}$ alcançando um equilíbrio entre o $\mathrm{CO}_{2}$ absorvido pela cavidade abdominal e o removido pela ventilação, após este período ocorre o armazenamento em reservatórios corporais ${ }^{22}$.

A monitorização da $\mathrm{P}_{\mathrm{ET}} \mathrm{CO}_{2}$ é imprescindível em cirurgias laparoscópicas. Em algumas situações, principalmente na presença de doenças respiratórias, a medida da $\mathrm{P}_{\mathrm{ET}} \mathrm{CO}_{2}$ pode não estar correlacionada com a $\mathrm{PaCO}_{2}$, devido à presença de shunt pulmonar, do aumento da PIAe de alterações produzidas pela anestesia. Nestas situações pode-se ter pequenas elevações da $\mathrm{P}_{\mathrm{ET}} \mathrm{CO}_{2}$ e grandes elevações na $\mathrm{PaCO}_{2}$, com um aumento no gradiente artério-alveolar a- $\mathrm{P}_{\mathrm{ET}}\left(\mathrm{PCO}_{2}\right)$. Amonitorização através da gasometria arterial é recomendada em pacientes com doença pulmonar ${ }^{26,27}$. Muitos estudos indicam que a diferença a- $\mathrm{P}_{\mathrm{ET}}\left(\mathrm{PCO}_{2}\right)$ tende a aumentar ou tornar-se imprevisível durante o tempo de aplicação do PPC, principalmente em pacientes e animais com disfunção pulmonar ${ }^{26,-31}$

Em pacientes com SARA, que possuem alterações da complacência e resistência pulmonares, foi observado melhor padrão respiratório com a $\mathrm{PCV}^{17}$

Apesar da PCV ter sido estudada comparativamente na presença de doenças pulmonares, não foi encontrado estudo comparativo sobre o uso da PCV e da VCV em anestesia com o uso de pneumoperitônio, onde a complacência e a resistência pulmonares sofrem alterações, mesmo que reversíveis ao fim do procedimento cirúrgico.

Diversos estudos demonstraram que a freqüência cardíaca não sofre alterações significativas durante o pneumoperitônio ${ }^{32-41}$.

O aumento da freqüência cardíaca foi similar nos dois grupos, após a aplicação do PPC, sem diferenças entre a ventilação com o controle do volume ou o controle da pressão. A maioria dos estudos relata um aumento na pressão arterial média (PAM) após a insuflação da cavidade abdominal para a criação de um pneumoperitônio ${ }^{33-49}$.

Alguns estudos observaram um aumento na concentração plasmática do hormônio antidiurético (ADH) após a criação do pneumoperitônio ${ }^{40,50,51}$, suficiente para determinar o aumento da PAM e da SVR por vasoconstrição $40,52,53$

Acriação do pneumoperitônio com a insuflação de $\mathrm{CO}_{2}$ leva a um aumento da concentração plasmática da renina ${ }^{54-56}$ e da aldosterona ${ }^{55}$ e da noradrenalina. Um estudo não observou alterações significativas nas concentrações da epinefrina, da norepinefrina e na atividade da renina plasmática, que pudessem estar correlacionadas ao aumento da PAM ${ }^{40}$. A hipercapnia pode levar a um aumento da PAM ${ }^{1,57}$. Neste estudo os valores da $\mathrm{PaCO}_{2}$ situaram-se dentro dos limites da normalidade o que pode ter contribuído para os valores da PAM.

Não houve diferença de comportamento da pressão arterial média entre os grupos VC e PC, apesar dos valores do grupo VC serem superiores aos do grupo PC desde o início do experimento e situarem-se dentro dos limites da normalidade.

Diversos estudos relatam aumento da pressão do átrio direito durante o uso do pneumoperitônio $37,41,49,58,59$.

Durante o PPC, o efeito mecânico da insuflação comprime os vasos de capacitância (sistema venoso) e os vasos de resistência (sistema arterial) levando a aumento significativo das pressões de enchimento cardíaco, nas câmaras esquerda e direita ${ }^{34,60,61 .}$.

O aumento da PIA leva a um aumento da pressão intratorácica ocasionando um aumento da pressão sobre as câmaras cardíacas, com aumento da pressão do átrio direito e da pressão da artéria pulmonar ocluida ${ }^{28,62}$

O aumento da PIA tem efeito bifásico sobre o retorno venoso com um aumento da compressão dos vasos de capacitância intra-abdominais, seguido da impedância do retorno venoso do abdômen para os membros inferiores. Durante o PPC, ocorre aumento da pressão das veias femorais com a diminuição do fluxo sangüíneo proveniente dos membros inferiores ${ }^{63}$.

Outros estudos realizados em porcos ${ }^{57}$ e em cães ${ }^{35,64}$ não observaram alterações nos valores da pressão de átrio direito, durante a aplicação do pneumoperitônio.

O controle da ventilação a volume ou a pressão não interferiu na pressão do átrio direito. Em todos os momentos os valores do grupo PC foram menores do que os valores do grupo VC, por uma provável característica do grupo dos animais. Em M4 esta diferença foi significativa, porém os valores observados situam-se dentro da faixa da normalidade.

Em um grupo de 16 pacientes, estado físico ASA I e II, 24 minutos após a insuflação do $\mathrm{CO}_{2}$, foi observada a presença de acidose respiratória moderada ${ }^{33}$ que pode ser causada parcialmente pela diminuição do débito cardíaco, que diminui a perfusão periférica, fato que pode ser agravado com a tentativa de aumento da ventilação, aumentando a depressão sobre o débito cardíaco, piorando a acidose. Pode ocorrer a necessidade de interromper um procedimento com PPC, após varias tentativas de corrigir a acidose com o aumento da ventilação ${ }^{26}$.

Um estudo dos efeitos ventilatórios, das alterações dos gases sangüíneos e do consumo de oxigênio durante histerectomias laparoscópicas, observou o desenvolvimento de acidose metabólica moderada durante a laparoscopia, mas após o procedimento a principal acidose foi de natureza respiratória $^{7}$.

$\mathrm{Na}$ análise estatística dos valores do $\mathrm{pH}$ arterial ficou demonstrado que ocorreu interação entre os grupos e os momentos, com perfis não similares. Apesar da maior variação dos valores do grupo VC, não houve diferenças entre grupos em cada momento. No grupo VC ocorreu uma diminuição dos valores a partir de $M 1$, sendo $M 1>(M 2=M 3=M 4)$. No grupo $\mathrm{PC}$ ocorreu uma diminuição dos valores a partir de $\mathrm{M} 1$, sendo que: $\mathrm{M} 1$ > M2 e (M3 = M4) com valores intermediários. Ape- 
sar da maior estabilidade nos valores do $\mathrm{pH}$ arterial na ventilação controlada a pressão não houve diferença estatística significativa entre os dois grupos.

$\mathrm{O}$ controle dos valores da $\mathrm{P}_{\mathrm{ET}} \mathrm{CO}_{2}$ no ar expirado foi um dos alvos de atenção deste estudo. Foram realizados ajustes, no volume corrente (grupo VC) e na pressão inspiratória (grupo $\mathrm{PC})$, para a manutenção da $\mathrm{P}_{\mathrm{ET}} \mathrm{CO}_{2}$ dentro dos limites pretendidos.

$\mathrm{O} \mathrm{CO}_{2}$ insuflado na cavidade abdominal leva a hipercarbia e acidose e deve ser removido através do aumento da ventilação, adequando-se o volume minuto ${ }^{65}$.

Os ajustes realizados na ventilação, que refletem sobre os valores da $\mathrm{P}_{\mathrm{ET}} \mathrm{CO}_{2}$, promovem alterações na $\mathrm{PaCO}_{2}$.

Mesmo com normoventilalação peri-operatória, no período pós-operatório, até uma hora após a retirada do PPC pode ocorrer a acidose respiratória e a hipercapnia, pelo $\mathrm{CO}_{2}$ acumulado $^{33,66}$.

Em condições normais a diferença entre a $\mathrm{PaCO}_{2}$ e a $\mathrm{P}_{\mathrm{ET}} \mathrm{CO}_{2}$ $\left(\mathrm{a}-\mathrm{P}_{\mathrm{ET}} \mathrm{P}\left(\mathrm{CO}_{2}\right)\right)$ é de 2 a $5 \mathrm{mmHg}{ }^{67,68}$.

Apesar de muitos fatores influenciarem sobre o gradiente a- $\mathrm{P}_{\mathrm{ET}} \mathrm{P}\left(\mathrm{CO}_{2}\right)$, ele é considerado como um índice do espaço morto $^{7}$

Um estudo com porcos submetidos ao PPC observou aumento do espaço morto com conseqüente diminuição da ventilação alveolar e aumento da $\mathrm{PaCO}_{2}$, se não houver correção do volume minuto ${ }^{24}$.

Neste estudo o gradiente a- $\mathrm{P}_{\mathrm{ET}} \mathrm{P}\left(\mathrm{CO}_{2}\right)$ medido em $\mathrm{mmHg}$, no grupo VC foi de 4,0 em M1, 4,7 em M2, 7,2 em M3 e 5,2 em M4, enquanto no grupo PC foi de 5,1 em M1, 3,6 em M2, 5,2 em M3 e 4,3 em M4. Estes valores demonstram menor gradiente alvéolo-arterial na ventilação controlada a pressão.

Vários estudos indicam que a diferença alvéolo-arterial tem uma tendência a aumentar com o tempo do PPC, tornando-se imprevisível em procedimentos prolongados, principalmente em pacientes com doença pulmonar, recomendando o uso da gasometria arterial para melhor avaliação da pressão do $\mathrm{CO}_{2}$ sangüíneo ${ }^{26,27,30,31}$. Outros estudos apontam o hélio como um gás alternativo por promover menores variações da diferença alvéolo-arterial, evitando a hipercapnia $24,27,69$

O volume de $\mathrm{CO}_{2}$ armazenado durante o procedimento pode levar a hipercapnia e acidose respiratória no período pós-operatório, de pacientes com hipoventilação ou débito cardíaco diminuído, como nos portadores de doença pulmonar ou doença cardíaca.

Neste estudo, as variações observadas na $\mathrm{PaCO}_{2}$ foram as mesmas observadas na $\mathrm{P}_{\mathrm{ET}} \mathrm{CO}_{2}$, com relação aos grupos e momentos, mesmo que com magnitudes diferentes. Ocorreu maior variação dos valores da $\mathrm{PaCO}_{2}$ no grupo VC, que aumentou proporcional ao aumento da PIA e apresentou diferenças estatísticas significativas em M2. Os controles sobre a ventilação (volume ou pressão) apresentaram diferenças sobre a $\mathrm{PaCO}_{2}$, sendo que a ventilação controlada a pressão apresentou maior linearidade.

O PPC produz um aumento do espaço morto que pode diminuir a ventilação alveolar, caso não haja o ajuste e controle da ventilação, principalmente na presença de doenças car- díaca e pulmonar e com baixos valores de $\mathrm{F}_{1} \mathrm{O}_{2}$ (fração inspiratória de oxigênio).

A compressão pulmonar com a diminuição da complacência e o aumento da pressão das vias aéreas podem levar ao barotrauma e ao pneumotórax, a distribuição inadequada da ventilação com a alteração da relação ventilação/perfusão e áreas de atelectasias. Apesar destas alterações a oxigenação eventualmente sofre comprometimento importante ${ }^{70}$. Outros estudos demonstram que $\mathrm{PaO}_{2}$ não se altera durante o PPC em adultos ${ }^{7,15,27}$.

Nesta pesquisa o controle da ventilação a volume ou a pressão não interferiu sobre a pressão parcial de oxigênio no sangue arterial.

Nas condições desta pesquisa a ventilação controlada a volume e a ventilação controlada a pressão não determinaram alterações hemodinâmicas significativas.

A ventilação controlada a pressão determinou maior estabilidade dos valores da $\mathrm{P}_{\mathrm{ET}} \mathrm{CO}_{2}$ e da $\mathrm{PaCO}_{2}$, bem como menor variação nos valores do $\mathrm{pH}$ do sangue arterial.

Foi possível o uso da ventilação controlada a pressão (PCV), para anestesias em cães submetidos a pneumoperitônio com $\mathrm{CO}_{2}$, sendo necessário o controle rigoroso da ventilação, de acordo com as variações da pressão intra-abdominal.

\section{Hemodynamic and Ventilatory Effects of Volume or Pressure Controlled Ventilation in Dogs Submitted to Pneumoperitoneum. Comparative Study}

Antonio Roberto Carraretto, TSA, M.D.; Pedro Thadeu Galvão Vianna, TSA, M.D.; Armando Vieira de Almeida, TSA, M.D.; Eliana Marisa Ganem, TSA, M.D.

\section{INTRODUCTION}

Videolaparoscopy is beneficial for patients and health systems and its use is increasing in number and complexity. It is necessary to expand the abdominal cavity in order to visualize the content and operate the instruments. Most common technique is carbon dioxide $\left(\mathrm{CO}_{2}\right)$ inflation to create pneumoperitoneum. Although the presence of $\mathrm{CO}_{2}$ in the metabolic system, absorption and increased blood concentration above certain values promotes cardiovascular effects such as increased cardiac output ${ }^{1,2}$, increased mean blood pressure, and increased plasma epinephrine and norepinephrine concentrations ${ }^{1}$. Its vasodilating effect may lead to systemic vascular resistance (SVR) decrease when not compensated by venous capacitance system through the sympathetic nervous system ${ }^{1,2}$. Reports on $\mathrm{CO}_{2}$ inhalation effects on the ventilatory mechanics of non-anesthetized individuals spontaneously breathing are controversial. Airways resistance may be increased ${ }^{3}$, decreased ${ }^{4}$ or unchanged $^{5}$. 
Increased intra-abdominal pressure has mechanical effects on abdomen and chest with cardiovascular and ventilatory changes, shifts the diaphragm in the cephalad direction, increases intra-thoracic pressure, lungs expansion is restricted by the shift of the abdominal part of the chest wall, dynamic pulmonary compliance decreases approximately $50 \%$, and Paw peak and Paw plat are increased ${ }^{6-8}$.

General anesthesia induces ventilatory changes, which need controlled ventilation. Most ventilators installed in anesthesia machines have volume-controlled ventilation (VC) and pressure limited ventilation (PLV). New imported and some domestic equipment are starting to incorporate pressure-controlled ventilation (PC) already present and common in Intensive Care Units (ICU). Adjusted parameters in VC are inspiratory volume and flow, both flow peak and shape. Ventilatory rate and the ratio between inspiratory and expiratory times (I:E ratio) are dependent on these adjustments.

Airways pressure is dependent on system's resistance and compliance. In LVP, adjusted parameters are limit pressure and inspiratory flow. Volume is dependent on resistance and compliance. Depending on the cycling mechanism, I:E ratio and respiratory rate may change. In PC, adjusted parameters are: airways pressure, inspiratory time or I:E ratio and ventilatory rate. These parameters affect inspiratory flow and tidal volume profiles, which should be monitored for adequate ventilation. Modern ICU ventilators have the PC modality and have been studied in acute respiratory distress syndrome (ARDS) patients.

Sometimes these patients are transferred from the ICU to the Operating Theater and there is a request to continue with the same ventilation modality. The described ventilation modalities allow the addition of positive end expiratory pressure (PEEP), to prevent or decrease collapse and improve alveolar recruitment. Hemodynamic ${ }^{9,10}$ and renal changes were evaluated by other studies with similar methods. This study aimed at evaluating the applicability of pressure-controlled ventilation (PC) in anesthesia in the presence of $\mathrm{CO}_{2}$ pneumoperitoneum, by measuring hemodynamic and ventilatory parameters and comparing them to conventional volume controlled ventilation (VC).

\section{METHODS}

After the Animal Research Ethics Committee, Faculdade de Medicina, Botucatu - UNESP approval, 16 adult mixed breed dogs of both genders, weighing 15 to $23 \mathrm{~kg}$, supplied by the Lab Animals Facility of the Botucatu Campus, UNESP were involved in this study. Animals were in fast for 12 hours with free access to water. Animals were randomly distributed in two groups of 8: VC group - volume controlled ventilation; and PC group - Pressure controlled ventilation (PC).

Experimental sequence: 1) Equipment check, test and adjustment (anesthesia machine, monitoring, infusion pumps, solutions heater, animal heater, gas inflator; 2) Recording of animals' weight, length and gender; 3) Anesthetic induction with intravenous sodium thiopental $\left(15 \mathrm{mg} \cdot \mathrm{kg}^{-1}\right)$, fentanyl

Revista Brasileira de Anestesiologia

Vol. 55, N 6, Novembro - Dezembro, 2005 $\left(0.015 \mathrm{mg} . \mathrm{kg}^{-1}\right)$ and pancuronium $\left.\left(0.07 \mathrm{mg} . \mathrm{kg}^{-1}\right) ; 4\right)$ Tracheal intubation ( $\mathrm{TI})$ with cuffed endotracheal tube $(8.5 \mathrm{~mm}$ ID) and checking; 5) Connection of Biomonitor Datex $A S 3^{\circledR}$ adapter to the $\mathrm{Y}$ connector of the $\mathrm{CO}_{2}$ absorber filter of the anesthesia machine Ohmeda Excel $210 \mathrm{SE}^{\circledR}$, with fresh gases flow (FGF) $=0.5 \mathrm{~L} \cdot \mathrm{min}^{-1}$ of $100 \%$ oxygen $\left(\mathrm{F}_{1} \mathrm{O}_{2}=1.0\right)$, to measure ventilation and analyze inspired and expired gases; 6 ) Beginning of mechanically controlled ventilation with Ohmeda 7900 ventilator coupled to the anesthesia machine in the selected modality, VC or PC, determined by the group, and adjustment of parameters to maintain desired ventilatory stability; 7) Installation of noninvasive monitoring: continuous ECG at $D_{\text {॥l lead, }}$ pulse oximeter and esophageal thermometer probe; 8) Right femoral vein dissection and catheterization for fluid and drug infusion and beginning of intravenous hydration with 6 $\mathrm{mL} . \mathrm{kg} \cdot \mathrm{h}^{-1}$ Ringer's solution, through infusion pump and heater with temperature control at $36.5^{\circ} \mathrm{C} ; 9$ ) left femoral vein dissection and catheterization to monitor mean blood pressure (MBP) and blood collection for blood gases analysis, connected to the measurement transducer of previously gauged Datex AS3 ${ }^{\circledR}$ monitor; 10) Right jugular vein dissection to introduce 7F Swan-Ganz catheter until the pulmonary artery for hemodynamic measures; 11) Median minilaparotomy to introduce $14 \mathrm{~F}$ polyethylene catheter for gas inflation and IAP control, with Olympus Surgical Inflator $15-\mathrm{L}^{\circledR}$ and gas removal at moment M3; 12) Animal covering with warming blanket by warm air inflation $\left(42-46^{\circ} \mathrm{C}\right)$ using WarmTouch ${ }^{\circledR}$ equipment.

Anesthesia was maintained with thiopental $\left(6 \mathrm{mg} \cdot \mathrm{kg}^{-1} \cdot \mathrm{h}^{-1}\right)$ and fentanyl $\left(0.006 \mathrm{mg} \cdot \mathrm{kg}^{-1} \cdot \mathrm{h}^{-1}\right)$ in continuous infusion with ANNE $^{\circledR}$ infusion pump. Additional pancuronium $(0.02$ $\left.\mathrm{mg} . \mathrm{kg}^{-1}\right)$ and fentanyl $\left(0.005 \mathrm{mg} \cdot \mathrm{kg}^{-1}\right)$ doses were administered when needed.

$\mathrm{CO}_{2}$ inflation in the abdominal cavity, to reach $10 \mathrm{mmHg}$ IAP (after M1) and $15 \mathrm{mmHg}$ (after M2) and gas removal for 0 mmHg IAP (after M3).

Animals were sacrificed at the end of experiment with $10 \mathrm{~mL}$ of intravenous $19.1 \%$ potassium chloride.

The following attributes were evaluated: weight $(\mathrm{kg})$, length $(\mathrm{cm})$ body surface (calculated $-\mathrm{m}^{2}$ ), gender (M/F), respiratory rate $\left(r-m_{0} \cdot \mathrm{min}^{-1}\right.$ tidal volume $\left(\mathrm{V}_{\mathrm{T}}-\mathrm{mL}\right)$, peak inspiratory pressure (Paw peek - $\left.\mathrm{cmH}_{2} \mathrm{O}\right)$, plateau inspiratory pressure (Paw plat $-\mathrm{cmH}_{2} \mathrm{O}$ ), chest compliance $\left(\mathrm{C}_{\mathrm{T}}-\mathrm{mL} \cdot \mathrm{cm}^{-1} \mathrm{H}_{2} \mathrm{O}\right)$, end tidal $\mathrm{CO}_{2}\left(\mathrm{P}_{\mathrm{ET}} \mathrm{CO}_{2}-\mathrm{mmHg}\right)$, heart rate $\left(\mathrm{HR}-\right.$ beat. $\left.\mathrm{min}^{-1}\right)$, mean blood pressure ( $\mathrm{MBP}-\mathrm{mmHg}$ ), mean right atrium pressure $(\mathrm{RAP}-\mathrm{mmHg})$, arterial $\mathrm{pCO}_{2}\left(\mathrm{PaCO}_{2}-\mathrm{mmHg}\right)$, and arterial $\mathrm{pO}_{2}\left(\mathrm{PaO}_{2}-\mathrm{mmHg}\right)$.

After catheter installation for CPP and monitoring, the 30-minute count was started looking for ventilatory stability by adjusting tidal volume (TV) or inspiratory pressure $(\underline{P C})$ to maintain $\mathrm{P}_{\mathrm{ET}} \mathrm{CO}_{2}$ within desired limits (35 to $45 \mathrm{mmHg}$ ), with respiratory rate regulated to $10 \mathrm{ipm} \cdot \mathrm{min}^{-1}, \mathrm{I}: \mathrm{E}$ ratio $=1: 2$ and PEEP of $3 \mathrm{cmH}_{2} \mathrm{O}$. Ventilatory measures were obtained with Datex $A S 3^{\circledR}$ monitor with the probe close to the tracheal tube connector. This procedure has been previously validated ${ }^{11}$. Ventilatory, hemodynamic, blood and temperature measures were collected in moments M1, M2, M3 and M4, being: M1 - 
immediately after the end of 30-minute stabilization period with IAP $=0 \mathrm{mmHg}$; M2 - 30 minutes after CPP with IAP = 10 $\mathrm{mmHg}$; $\mathrm{M} 3$ - 30 minutes after CPP with IAP $=15 \mathrm{mmHg}$; M4 30 minutes after CPP with IAP $=0 \mathrm{mmHg}$.

Analysis of Variance was used for weight, length and body surface area to compare between groups.

Profile analysis was used for remaining variables evaluated in the four moments ${ }^{13}$.

\section{RESULTS}

Weight, length and body surface area were not significantly different between groups and are shown in table I.

Table I - Demographics Data (Mean \pm SD)

\begin{tabular}{lccl}
\hline Groups & Weight $(\mathrm{kg})$ & Length $(\mathrm{cm})$ & BSA $\left(\mathrm{m}^{2}\right)$ \\
\hline $\mathrm{VC}(\mathrm{n}=8)$ & $20.3 \pm 3.1$ & $105.1 \pm 8.0$ & $0.8 \pm 0.1$ \\
$\mathrm{PC}(\mathrm{n}=8)$ & $18.6 \pm 1.5$ & $100.3 \pm 4.1$ & $0.7 \pm 0$ \\
\hline
\end{tabular}

Tidal volumes, regulated to maintain $\mathrm{P}_{\mathrm{ET}} \mathrm{CO}_{2}$ between 35 and $45 \mathrm{mmHg}$ were not significantly different between groups in each moment. Values were constant for the VC group and increased for $\mathrm{PC}$ group after pneumoperitoneum, not returning to baseline values (Figure 1).

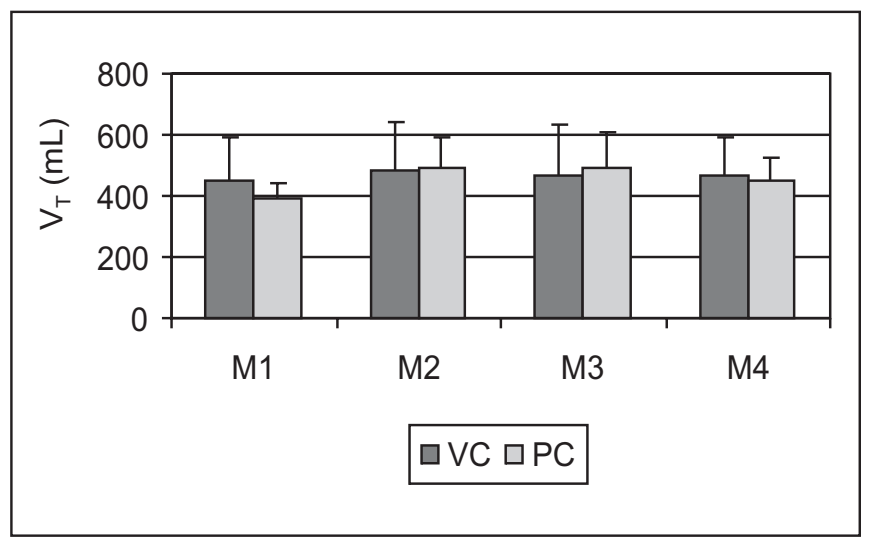

Figure 1 - Tidal Volume $(\mathrm{mL})$ of Studied Groups and Moments (Mean \pm SD)

Peak inspiratory pressure increased with IAP increase for both groups, with a trend to higher values for VC group in $\mathrm{M} 1$ and $\mathrm{M} 4$, and returning to baseline values after deflation (Figure 2).

Plateau inspiratory pressure has increased with IAP increase and has returned to baseline values after deflation, with similar behavior between groups (Figure 3).

Compliance values have decreased with increased IAP in both groups. After deflation, VC group has returned to baseline values and PC group has remained with higher values (Figure 4).

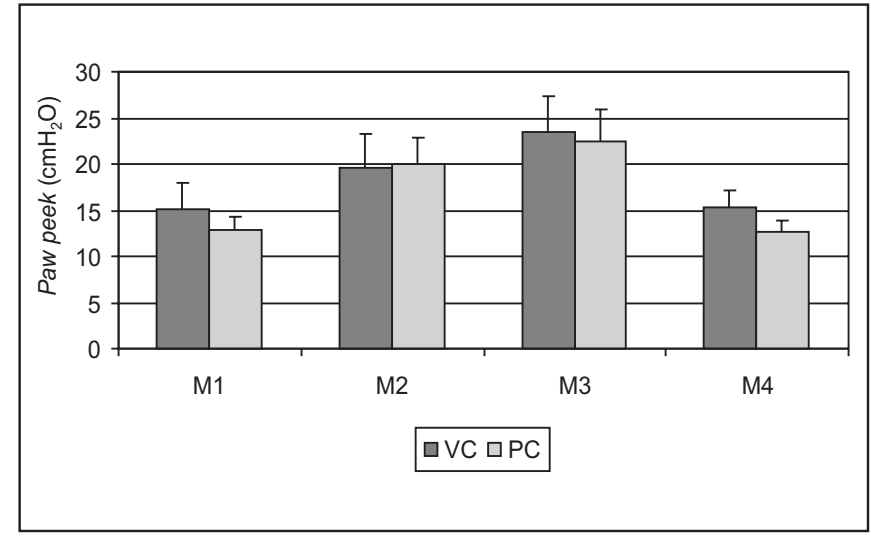

Figure 2 - Peak Inspiratory Pressure $\left(\mathrm{cmH}_{2} \mathrm{O}\right)$ of Studied Groups and Moments (Mean \pm SD)

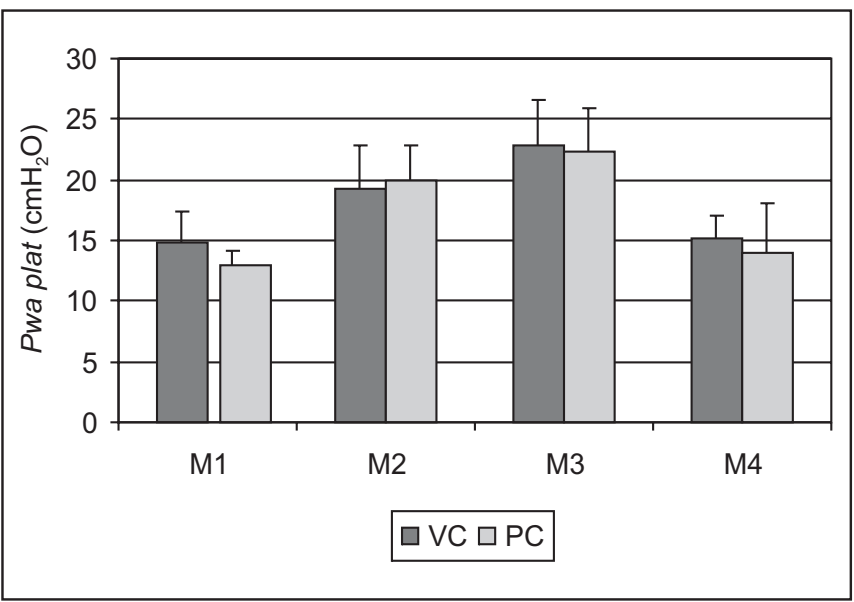

Figure 3 - Plateau Inspiratory Pressure $\left(\mathrm{cmH}_{2} \mathrm{O}\right)$ of Studied Groups and Moments (Mean \pm SD)

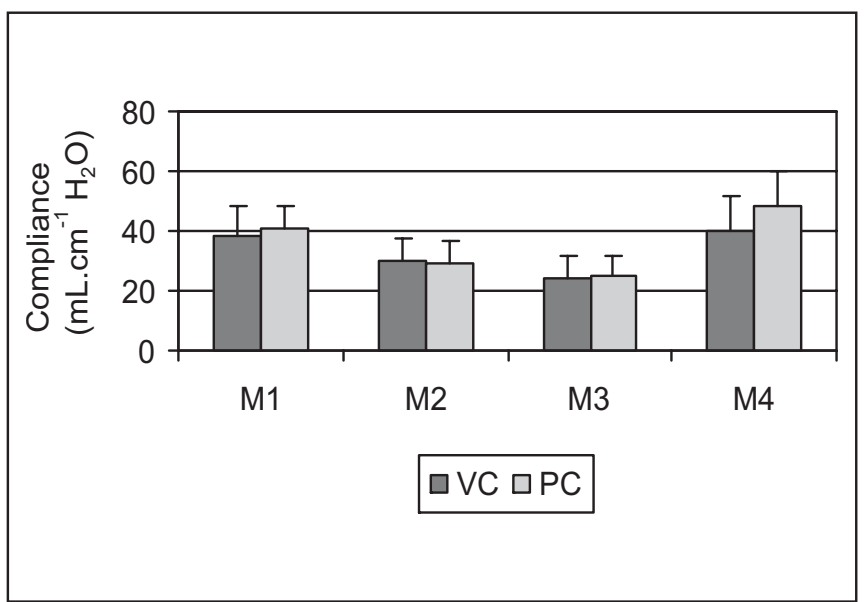

Figure 4 - Compliance $\left(\mathrm{mL} . \mathrm{cm}^{-1} \mathrm{H}_{2} \mathrm{O}\right)$ of Studied Groups and Moments (Mean \pm SD)

Revista Brasileira de Anestesiologia Vol. 55, N 6 , Novembro - Dezembro, 2005 
End tidal $\mathrm{CO}_{2}$ was higher for VC group as compared to PC group during pneumoperitoneum, returning to baseline values after deflation. $\mathrm{PC}$ group presented greater uniformity of values (Figure 5).

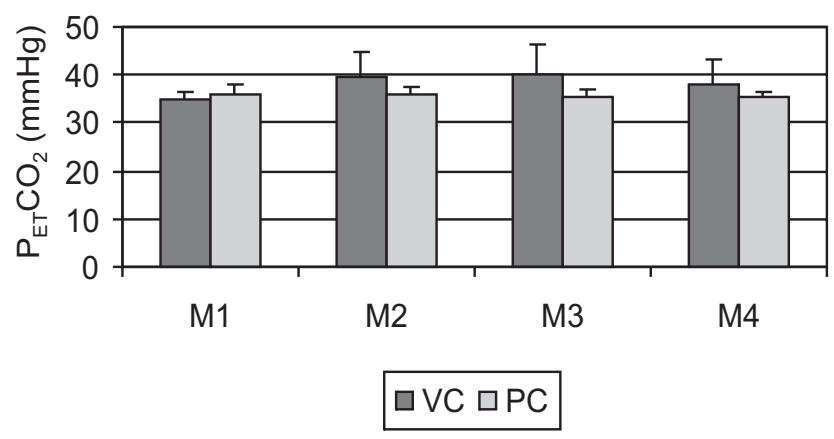

Figure $5-\mathrm{pCO}_{2}$ of Studied Groups and Moments (Mean \pm SD)

Heart rate had the same behavior for both groups, with higher values in M2 and M3 (Figure 6).

Mean blood pressure was higher for VC group as compared to $\mathrm{PC}$ group. There were no differences among moments for both groups (Figure 7).

Right atrium pressure has increased during pneumoperitoneum and has decreased after deflation, with significant decrease in PC group (Figure 8).

Blood $\mathrm{pH}$ was different among moments. In VC group it decreased during pneumoperitoneum. In PC group it had less variation (Figure 9).

Arterial $\mathrm{pCO}_{2}$ was different between groups with increase (M2) and trend to increase (M3) in VC group during pneumoperitoneum, and stability in PC group (Figure 10).

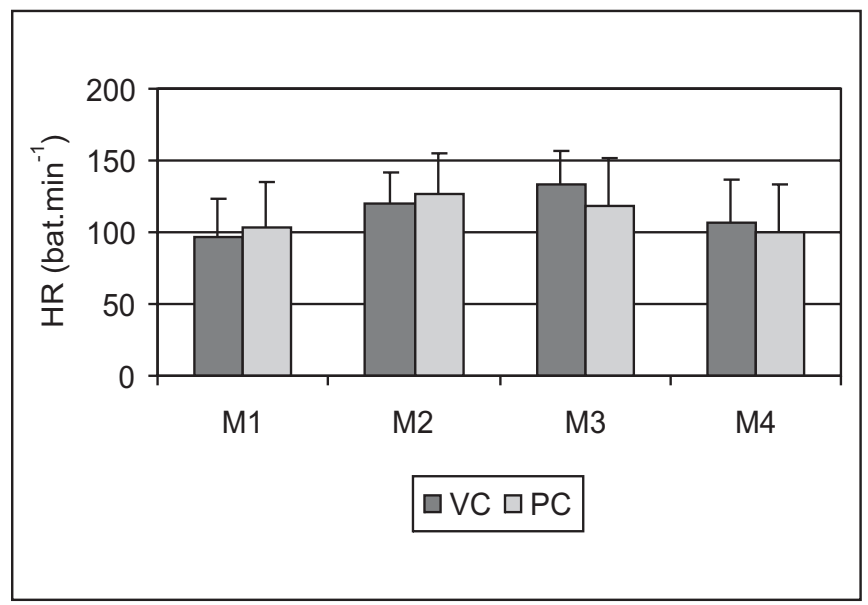

Figure 6 - Heart Rate (beat.min ${ }^{-1}$ ) of Studied Groups and Moments $($ Mean $\pm \mathrm{SD})$

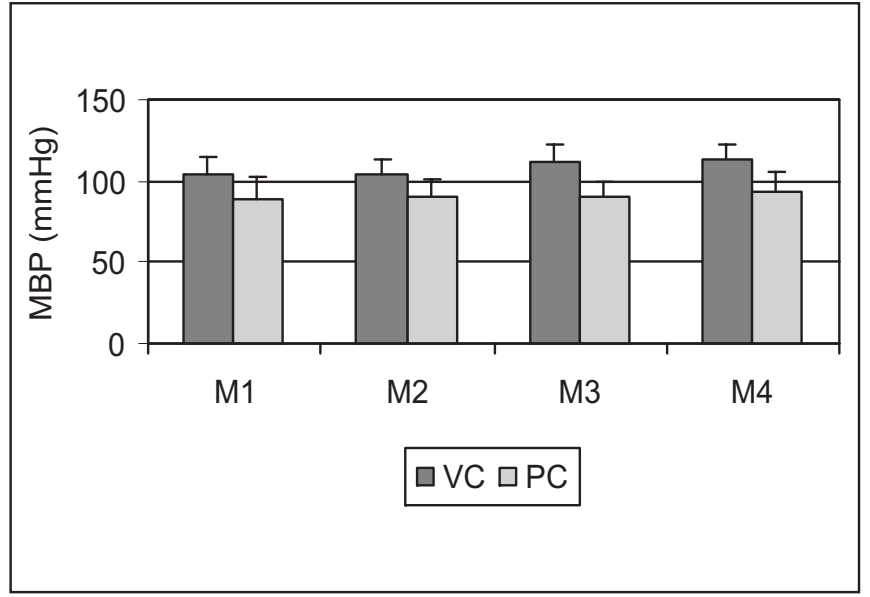

Figure 7 - Mean Blood Pressure $(\mathrm{mmHg})$ of Studied Groups and Moments (Mean $\pm \mathrm{SD}$ )

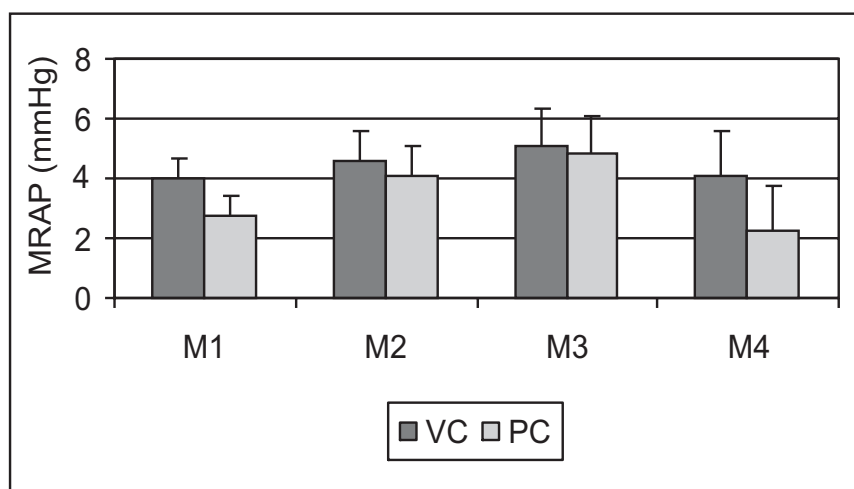

Figure 8 - Mean Right Atrium Pressure $(\mathrm{mmHg})$ of Studied Groups and Moments (Mean \pm SD)

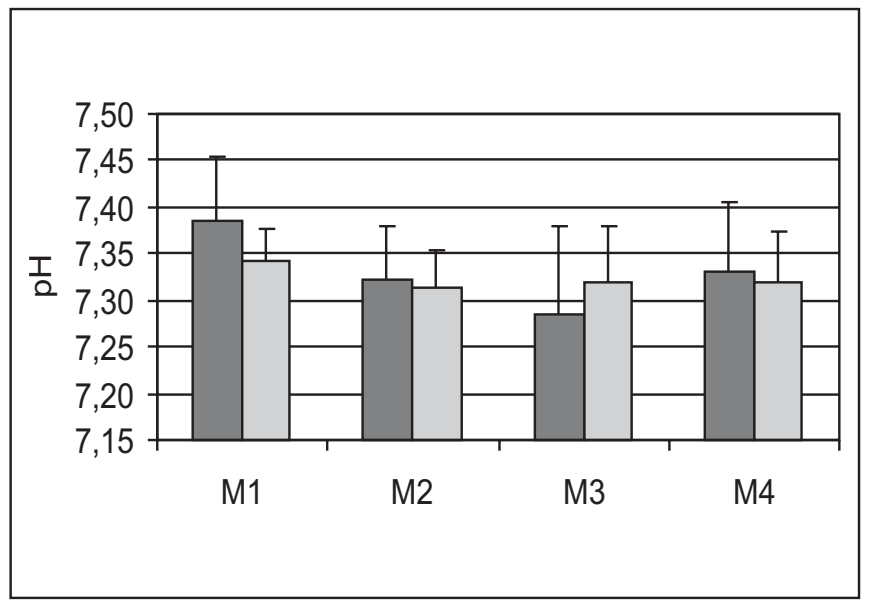

Figure 9 - Arterial pH of Studied Groups and Moments (Mean \pm SD) 
Arterial $\mathrm{pO}_{2}$ had the same behavior, without differences between groups and moments (Figure 11).

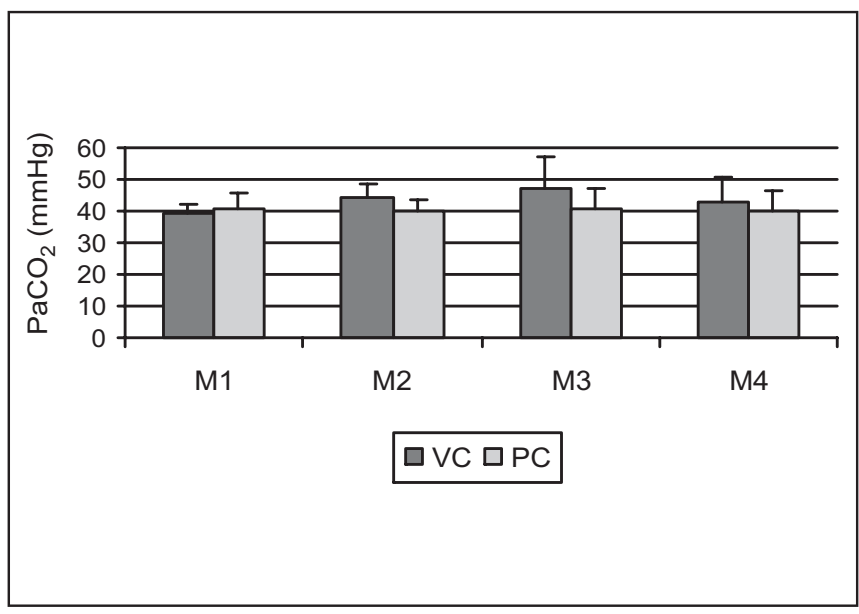

Figure 10 - Arterial $\mathrm{pCO}_{2}(\mathrm{mmHg})$ of Studied Groups and Moments $($ Mean \pm SD)

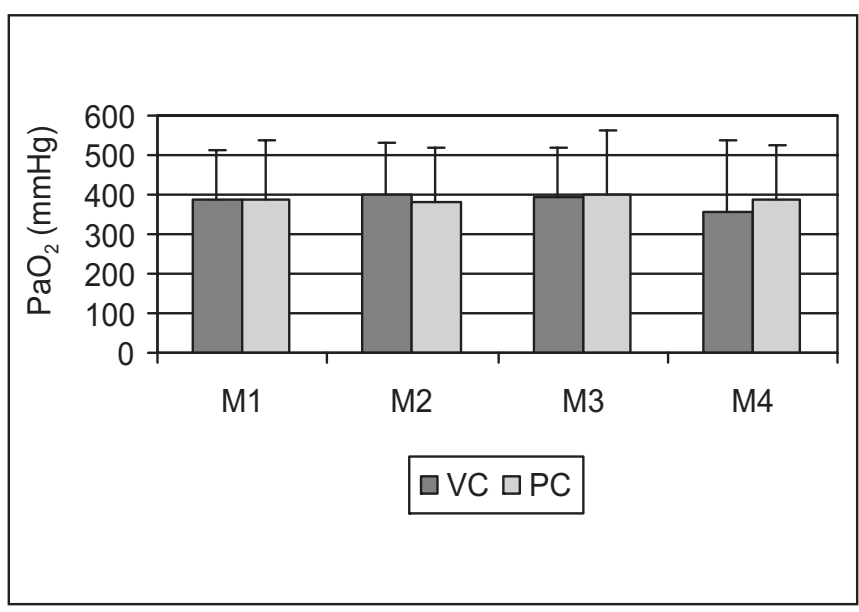

Figure 11 - Arterial $\mathrm{pO}_{2}(\mathrm{mmHg})$ of Studied Groups and Moments $($ Mean \pm SD)

\section{DISCUSSION}

Among major available controls during mechanically controlled ventilation there are volume and pressure, object of our study. These may be individually or jointly preset, generating volume controlled ventilation (VCV) or pressure controlled ventilation (PCV). Control combinations, such as target volume, pressure controlled and time cycling are among new available modalities called double control modes. In our study, to better control different evaluated parameters, respiratory rate was established in 10 mov. $\mathrm{min}^{-1}$, similar to other studies $^{7,14}$, and I:E ratio in 1:2. These values allowed adequate animals ventilation, confirmed by values obtained from $\mathrm{P}_{\mathrm{ET}} \mathrm{CO}_{2}, \mathrm{PaCO}_{2}$ and $\mathrm{PaO}_{2}$ analyses.
Tidal volume had to be increased by adjusting volume control in VC group or pressure control in PC group, to maintain $\mathrm{P}_{\mathrm{ET}} \mathrm{CO}_{2}$ within desired values. A different study has shown the need to increase respiratory minute volume, from $20 \%$ to $30 \%$, to eliminate absorbed $\mathrm{CO}_{2}$ by CPP ${ }^{15}$.

In VCV, the volume to be administered is a fixed parameter (predetermined) together with ventilatory rate and I:E ratio. The ventilator generates a controlled flow, which is interrupted at the end of inspiration. After it, there may be an inspiratory pause (with maintenance of plateau pressure) or the cycling to the expiratory phase. Pressure will be the result of ventilatory mechanics, such as changes on resistance and compliance. With increased IAP, increased intra-thoracic pressure increases airways pressure. Minute volume suffers minor or no change and alveolar ventilation, responsible for alveolar $\mathrm{CO}_{2}$ removal, is maintained.

In PCV, the ventilator starts the cycle with a demand flow needed to maintain a predetermined pressure (fixed parameter). When this pressure is reached, the flow is gradually decreased (slow down flow) to maintain the pressure. Tidal volume depends on regulated pressure, inspiratory time and, as in any pressure mode, will be influenced by ventilatory mechanics changes (compliance and resistance) ${ }^{16}$.

During PCV, at inspiratory valve opening, gas flow is high and most volume is distributed in the beginning of the inspiratory phase. Pressure control valve regulates the inspiratory flow maintaining the pressure constant e allowing a better gas distribution to alveoli with higher time constant without promoting hyperdistension - by controlling alveolar pressure. Increased ventilatory rate or inspiratory pressure are needed to improve alveolar ventilation

Increased airways resistance or decreased pulmonary compliance decrease tidal volume and, as a consequence, alveolar ventilation. Due to IAP effects on intra-thoracic pressure, each IAP variation may need correction of ventilatory parameters, in general a new pressure, to compensate the effect of tidal volume loss or gain.

In a comparative study between PCV and VCV, in patients with severe acute respiratory distress syndrome (ARDS), it has been observed less cardiac output decrease in the PCV group, reported as the result of right ventricle overload improvement due to better alveolar recruitment, decreased pulmonary vascular resistance and decreased intra-thoracic pressure, which has helped a better preload of both ventricles ${ }^{17}$.

A different comparative study with patients submitted to single-lung ventilation has observed significantly higher Paw peak, Paw plat and pulmonary shunt during VCV, while $\mathrm{PaCO}_{2}$ was higher during PCV. The study has concluded that $P C V$ is an alternative to VCV in patients needing single-lung ventilation, and may be better than VCV in respiratory disease patients ${ }^{18}$.

In the postoperative period of cardiac surgeries, both controls had comparable effects in patients with preserved or depressed cardiac function, but PCV patients showed higher cardiac index values, decreased SVR and lower inspiratory pressure values, as compared to VCV patients ${ }^{19}$. Vol. 55, № 6 , Novembro - Dezembro, 2005 
PCV has been used in ICU to treat patients with severe respiratory diseases, with better results on oxygenation and better prevention of pulmonary tissue injury ${ }^{20}$. These patients, already with cardiovascular and/or respiratory disorders, when submitted to procedures requiring CPP, are more difficult to have their ventilation controlled and normocapnia and oxygenation maintained. There are situations needing surgeries with pneumoperitoneum in patients already using PCV or when this modality is the best indication.

A major PCV characteristic is the dependence among patient's respiratory mechanics, inspiratory flow and volume. $\mathrm{PCV}$ prevents high pressures, determining the mechanism of pulmonary injury in the presence of differences in alveolar resistance and compliance, by maintaining airway pressure constant $^{21}$.

Maximum absorption is seen with relatively low pressures of $10 \mathrm{mmHg}$. Absorbed gas is eliminated by the lungs, sometimes even in the postoperative period, since volumes of more than 120 liters may be stored in our body. Bones are major reservoirs ${ }^{22}$.

In pressure controlled ventilation there has been the need to increase tidal volume after pneumoperitoneum.

Peak inspiratory pressure is the maximum generated pressure to inflate lungs. It depends on the respiratory system elastic and resistive properties. Elastic components generate the pressure needed to change pulmonary volume while resistive properties represent the necessary pressure to generate gas flow ${ }^{16}$.

There has been a proportional Paw Peak increase during CPP, without significant difference between VC and PC control modes.

CPP has determined proportional Paw plat increase, which has returned to baseline values after removal, without significant difference between volumes controlled and pressure controlled ventilation.

End tidal $\mathrm{CO}_{2}$ measured by capnometry was controlled by our study. To maintain $\mathrm{P}_{\mathrm{ET}} \mathrm{CO}_{2}$ within desired limits, volume was adjusted for VC group and inspiratory pressure was adjusted for PC group.

It has been observed that during prolonged $\mathrm{CO}_{2}$ abdominal inflation, peritoneal absorption leads to increase in blood concentration and elimination. The pattern is characterized by an early phase with fast increase in $\mathrm{CO}_{2}$ elimination (27-37 mL. $\mathrm{min}^{-1}$ ) soon after beginning of inflation, followed by a slower phase due to peritoneal surface distention with compression of peritoneal vessels ${ }^{23}$. This two-phase pulmonary elimination pattern was also observed in animal models ${ }^{24}$.

$\mathrm{CO}_{2}$ is highly soluble in blood generating an acid solution. To prevent hypercapnia and respiratory acidosis, ventilation should be increased in approximately $20 \%$ to $30 \%{ }^{15,22}$.

During CPP, $\mathrm{P}_{\mathrm{ET}} \mathrm{CO}_{2}$ is progressively increased with time, reaching its peak 40 minutes later, if ventilation is maintained constant ${ }^{25}$, reaching a balance between $\mathrm{CO}_{2}$ absorbed by the abdominal cavity and that removed by ventilation. After this period there is storage in body reservoirs ${ }^{22}$.
$\mathrm{P}_{\mathrm{ET}} \mathrm{CO}_{2}$ monitoring is critical during laparoscopic surgeries. In some situations, especially in the presence of respiratory diseases, $\mathrm{P}_{\mathrm{ET}} \mathrm{CO}_{2}$ measure may not be correlated to that of $\mathrm{PaCO}_{2}$, due to the presence of pulmonary shunt, increased IAP and anesthesia-induced changes. In such situations, there may be minor $\mathrm{P}_{\mathrm{ET}} \mathrm{CO}_{2}$ increase and major $\mathrm{PaCO}_{2}$ increase, with increased arterial-alveolar gradient (a-P $\mathrm{P}_{\mathrm{ET}}$ $\left.\left(\mathrm{PCO}_{2}\right)\right)$. Monitoring with blood gases analysis is recommended for patients with pulmonary disease.

Several studies indicate that the difference a- $\mathrm{P}_{\mathrm{ET}}\left(\mathrm{PCO}_{2}\right)$ tends to increase or to become unforeseeable during CPP, especially in patients and animals with pulmonary dysfunction.

In ARDS patients, with changes in pulmonary compliance and resistance, a better respiratory pattern was observed with PCV ${ }^{17}$.

Although PCV has been comparatively studied in the presence of pulmonary diseases, no comparative study was found on PCV and VCV in anesthesia with pneumoperitoneum, where pulmonary compliance and resistance are changed, even if reversible at the end of surgery.

Several studies have shown that heart rate is not significantly changed during pneumoperitoneum ${ }^{32-41}$.

Heart rate increase was similar for both groups after CPP without differences between volume controlled or pressure controlled ventilation.

Most studies report increased mean blood pressure (MBP) after abdominal cavity inflation for pneumoperitoneum ${ }^{33-49}$.

Some studies have observed increased plasma concentration of antidiuretic hormone $(A D H)$ after pneumoperitoneum $40,50,51$, enough to determine increased MBP and SVR by vasoconstriction ${ }^{40,52,53}$.

$\mathrm{CO}_{2}$ pneumoperitoneum leads to increased rennin ${ }^{54-56}$, aldosterone ${ }^{55}$ and norepinephrine plasma concentration. A study has not observed significant epinephrine and norepinephrine concentrations, and plasma rennin activity changes which could be related to increased MBP ${ }^{40}$. Hypercapnia may increase $\mathrm{MBP}^{1,57}$. In our study, $\mathrm{PaCO}_{2}$ values were within normality ranges, which may have contributed to MBP values.

There was no difference in mean blood pressure behavior between VC and PC groups, although VC group values were higher as compared to PC group since the beginning of the experiment, however within normality ranges.

Several studies have reported increased right atrium pressure during pneumoperitoneum ${ }^{37,41,49,58,59}$.

During CPP, the mechanical inflation effect compresses capacitance vessels (venous system) and resistance vessels (arterial system) leading to significant increase in filling pressures in both left and right chambers ${ }^{34,60,61}$.

IAP increase leads to intra-thoracic pressure increase with consequent pressure increase in cardiac chambers, with increased right atrium pressure and pulmonary capillary wedge pressure ${ }^{28,62}$.

IAP increase has a two-phase effect on venous return, with increased compression of intra-abdominal capacitance vessels, followed by the impedance of abdominal venous re- 
turn to lower limbs. During CPP, there is increased femoral veins pressure with decreased blood flow coming from lower limbs ${ }^{63}$

Other studies with swine ${ }^{57}$ and dogs ${ }^{35,64}$ have not observed changes in right atrium pressure during pneumoperitoneum. Volume or pressure controlled ventilation has not interfered with right atrium pressure. In all moments, PC group values were lower as compared to VC group, maybe due to a possible characteristic of the group of animals. In M4 the difference was significant, however values were within normal ranges. In a group of 16 patients physical status ASA I and II, there has been moderate respiratory acidosis 24 minutes after $\mathrm{CO}_{2}$ inflation ${ }^{33}$, which might have been partially caused by decreased cardiac output, which decreases peripheral perfusion, which may be worsened by the attempt to increase ventilation, which increase cardiac output depression, worsening acidosis. CPP may have to be interrupted after several attempts to correct acidosis with increased ventilation ${ }^{26}$.

A study on ventilatory effects, blood gases changes and oxygen consumption during laparoscopic hysterectomies has observed the development of metabolic acidosis during laparoscopy, but after the procedure major acidosis was respiratory acidosis ${ }^{7}$

Statistical analysis of arterial $\mathrm{pH}$ has shown interaction between groups and moments, with different profiles. Although higher variation in VC group values, there were no differences between groups in each moment. VC group had decreased values as from $M 1$, being $M 1>(M 2=M 3=M 4) . P C$ group had decreased values as from $M 1$, being $M 1>M 2$ e $(M 3$ $=$ M4) with intermediate values. Although the higher stability of arterial $\mathrm{pH}$ values during pressure controlled ventilation, there were no statistically significant differences between groups.

$\mathrm{P}_{\mathrm{ET}} \mathrm{CO}_{2}$ in expired air was a target of attention in this study. Tidal volume (VC group) and inspiratory pressure (PC group) were adjusted to maintain $\mathrm{P}_{\mathrm{ET}} \mathrm{CO}_{2}$ within desired ranges.

$\mathrm{CO}_{2}$ inflated in the abdominal cavity leads to hypercabia and acidosis and should be removed by increased ventilation, adjusting minute volume ${ }^{65}$.

Ventilation adjustments, which affect $\mathrm{P}_{E T} \mathrm{CO}_{2}$ values, promote $\mathrm{PaCO}_{2}$ changes.

Even with normal perioperative ventilation, there may be respiratory acidosis and hypercapnia up to one hour after CPP removal, due to built-up $\mathrm{CO}_{2}{ }^{33,66}$.

In normal conditions, the difference between $\mathrm{PaCO}_{2}$ and $\mathrm{P}_{\mathrm{ET}} \mathrm{CO}_{2}\left(\mathrm{a}-\mathrm{P}_{\mathrm{ET}} \mathrm{P}\left(\mathrm{CO}_{2}\right)\right)$ is 2 to $5 \mathrm{mmHg}{ }^{67,68}$.

Although there are many factors influencing a- $\mathrm{P}_{\mathrm{ET}} \mathrm{P}\left(\mathrm{CO}_{2}\right)$ gradient, it is considered a dead space index.

Astudy with swine submitted to CPP has observed increased dead space with consequent alveolar ventilation decrease and $\mathrm{PaCO}_{2}$ increase, if minute volume is not corrected ${ }^{24}$. In our study, a- $\mathrm{P}_{\mathrm{ET}} \mathrm{P}\left(\mathrm{CO}_{2}\right)$ gradient, measured in $\mathrm{mmHg}$, in VC group was 4.0 in $M 1,4.7$ in $M 2,7.2$ in $M 3$ and 5.2 in $M 4$, while in PC group it was 5.1 in M1, 3.6 in M2, 5.2 in M3 and 4.3 in M4. These values show a lower alveolar-arterial gradient for pressure-controlled ventilation.
Several studies indicate that alveolar-arterial difference tends to increase with CPP time, becoming unforeseeable for prolonged procedures, especially in patients with pulmonary disease, and blood gases analysis is recommended for a better evaluation of arterial $\mathrm{pCO}_{2} 26,27,30,31$. Other studies point to helium as an alternative for promoting lower variations in alveolar-arterial difference, thus preventing hypercapnia $24,27,69$

$\mathrm{CO}_{2}$ volume stored during the procedure may lead to hypercapnia and respiratory acidosis in the postoperative period of patients with hypoventilation or decreased cardiac output, as well as in patients with pulmonary or cardiac disease. In our study, $\mathrm{PaCO}_{2}$ variations were the same observed in $\mathrm{P}_{\mathrm{ET}} \mathrm{CO}_{2}$ with regard to groups and moments, even if with different magnitudes. There have been higher $\mathrm{PaCO}_{2}$ variations in the VC group, which has increased as a function of $I A P$, increase and presented statistically significant differences in M2. Ventilation controls (volume or pressure) affected $\mathrm{PaCO}_{2}$ with more linearity for pressure-controlled ventilation.

CPP increases dead space and may decrease alveolar ventilation if there is no ventilation control adjustment, especially in the presence of cardiac and pulmonary disease and with low $\mathrm{FiO}_{2}$ (oxygen inspiratory fraction) values.

Pulmonary compression with decreased compliance and increased airway pressure may lead to barotrauma and pneumothorax, to the inadequate distribution of ventilation with changes in ventilation / perfusion ratio, and to atelectasis. In spite of these changes, oxygenation may suffer major impairment ${ }^{70}$.

Other studies have shown that $\mathrm{PaO}_{2}$ is not changed during CPP in adults ${ }^{7,15,27}$.

In our study, volume and pressure controlled ventilation have not affected arterial $\mathrm{pO}_{2}$.

In the conditions of our study, volume controlled and pressure controlled ventilation had not determined significant hemodynamic changes.

Pressure controlled ventilation has determined higher $\mathrm{P}_{\mathrm{ET}} \mathrm{CO}_{2}$ and $\mathrm{PaCO}_{2}$ stability, as well as less variations in arterial $\mathrm{pH}$.

Pressure controlled ventilation (PCV) was adequate to anesthetize dogs submitted to $\mathrm{CO}_{2}$ pneumoperitoneum, being necessary a strict ventilation control according to intra-abdominal pressure variations.

\section{REFERÊNCIAS - REFERENCES}

01. Rasmussen JP, Dauchot PJ, DePalma RG et al - Cardiac function and hypercarbia. Arch Surg, 1978;113:196-200.

02. Marshall RL, Jebson PJ, Davie IT et al - Circulatory effects of peritoneal insufflation with nitrous oxide. $\mathrm{Br} \mathrm{J}$ Anaesth, 1972;44:1183-1187.

03. Sterling GM - The mechanism of decreased specific airway conductance in man during hypercapnia caused by inhalation of $7 \%$ $\mathrm{CO}_{2}$, Clin Sci, 1969;37:539-548. 
04. Badr MS, Skatrud JB, Simon PM et al - Effect of hypercapnia on total pulmonary resistance during wakefulness and during NREM sleep. Am Rev Respir Dis, 1991;144:406-414.

05. Rodarte JR, Hyatt RE - Effect of acute exposure to $\mathrm{CO}_{2}$ on lung mechanics in normal man. Respir Physiol, 1973;17:135-145.

06. Bardoczky GI, Engelman E, Levarlet M et al - Ventilatory effects of pneumoperitoneum monitored with continuous spirometry. Anaesthesia, 1993:48:309-311.

07. Hirvonen EA, Nuutinen LS, Kauko M - Ventilatory effects, blood gas changes and oxygen consumption during laparoscopic hysterectomy. Anesth Analg, 1995;80:961-966.

08. Makinen MT, Yli-Hankala A - The effect of laparoscopic cholecystectomy on respiratory compliance as determined by continuous spirometry. J Clin Anesth, 1996;8:119-122.

09. Almeida AV, Ganem EM, Carraretto AR et al - Alterações hemodinâmicas durante o pneumoperitônio em cães ventilados com volume e pressão controlados. Rev Bras Anestesiol, 2003;53:756-766.

10. Almeida AV, Ganem EM - Efeitos do pneumoperitônio sobre a hemodinâmica e função renais em cães ventilados com volume e pressão controlados. Rev Bras Anestesiol, 2004;54:343-360.

11. Merilainen $P$, Hanninen $H$, Tuomaala $L$ - A novel sensor for routine continuous spirometry of intubated patients. J Clin Monit, 1993;9:374-380.

12. Curi PR - Metodologia e Análise da Pesquisa em Ciências

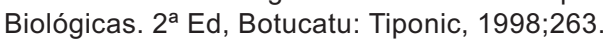

13. Morrison DF - Multivariate Statistical Methods, New York: McGraw Hill, 1967.

14. Fahy BG, Barnas GM, Flowers JL et al - The effects of increased abdominal pressure on lung and chest wall mechanics during laparoscopic surgery. Anesth Analg, 1995;81:744-750.

15. Tan PL, Lee TL, Tweed WA - Carbon dioxide absorption and gas exchange during pelvic laparoscopy. Can J Anaesth, 1992;39: 677-681.

16. David CM, Goldwasser R - Como Iniciar e Manter o Paciente em Ventilação Mecânica, em: David CM - Ventilação Mecânica: da Fisiologia à Prática Clínica. 2001;23:291-306.

17. Abraham E, Yoshihara G - Cardiorespiratory effects of pressure controlled ventilation in severe respiratory failure. Chest, 1990;98:1445-1449.

18. Tugrul M, Camci E, Karadeniz $\mathrm{H}$ et al - Comparison of volume controlled with pressure controlled ventilation during one-lung anaesthesia. Br J Anaesth, 1997;79:306-310.

19. Auler Junior JO, Carmona MJ, Silva MH et al - Haemodynamic effects of pressure-controlled ventilation versus volume-controlled ventilation in patients submitted to cardiac surgery. Clin Intensive Care, 1995;6:100-106.

20. Rappaport SH, Shpiner R, Yoshihara G et al - Randomized, prospective trial of pressure-limited versus volume-controlled ventilation in severe respiratory failure. Crit Care Med, 1994;22: 22-32.

21. Bonasa J - Princípios Básicos dos Ventiladores Artificiais, em: Carvalho CRR - Ventilação Mecânica: Básico. São Paulo, Atheneu, 2000;4:69-124.

22. Koivusalo AM, Lindgren L - Effects of carbon dioxide pneumoperitoneum for laparoscopic cholecystectomy. Acta Anaesthesiol Scand, 2000;44:834-841.

23. Mullett CE, Viale JP, Sagnard PE et al - Pulmonary $\mathrm{CO}_{2}$ elimination during surgical procedures using intra- or extraperitoneal $\mathrm{CO}_{2}$ insufflation. Anesth Analg, 1993;76:622-626.

24. Lister DR, Rudston-Brown B, Warriner CB et al - Carbon dioxide absorption is not linearly related to intraperitoneal carbon dioxide insufflation pressures in pigs. Anesthesiology, 1994;80: 129-136.
25. Baraka A, Jabbour S, Hammoud R et al - End-tidal carbon dioxide tension during laparoscopic cholecystectomy. Correlation with the baseline value prior to carbon dioxide insufflation. Anaesthesia, 1994;49:304-306.

26. Wittgen C, Andrus C, Fitzgerald $S$ et al - Analysis of the hemodynamic and ventilatory effects of laparoscopic cholecystectomy. Arch Surg, 1991;126:997-1001.

27. Fitzgerald S, Andrus C, Baudendistel L et al - Hypercarbia during carbon dioxide pneumoperitoneum. Am J Surg, 1992;163: 186-190.

28. Joris $\mathrm{J}$, Cigarini I, Legrand $\mathrm{M}$ et al - Metabolic and respiratory changes after cholecystectomy performed via laparotomy or laparoscopy. Br J Anaesth, 1992;69:341-345.

29. Monk TG, Weldon BC, Lemon D - Alterations in pulmonary function during laparoscopic surgery .Anesth Analg, 1993;76:S274.

30. Wahba RW, Mamazza J - Ventilatory requirements during laparoscopic cholecystectomy. Can J Anaesth, 1993;40:206-210.

31. Feig BW, Berger DH, Dougherty TB et al - Pulmonary effects of $\mathrm{CO}_{2}$ abdominal insufflation (CAI) during laparoscopy in high-risk patients. Anesth Analg, 1994;78:S108.

32. Hirvonen EA, Nuutinen LS, Kauko M - Hemodynamic changes due to Trendelenburg positioning and pneumoperitoneum during laparoscopic hysterectomy. Acta Anaesthesiol Scand, 1995;39:949-955.

33. Critchley LA, Critchley JA, Gin T - Haemodynamic changes in patients undergoing laparoscopic cholecystectomy: measurement by transthoracic electrical bioimpedance. $\mathrm{Br} \mathrm{J}$ Anaesth, 1993;70:681-683.

34. Joris JL, Noirot DP, Legrand MJ et al - Hemodynamic changes during laparoscopic cholecystectomy. Anesth Analg, 1993;76: 1067-1071.

35. Williams MD, Murr PC - Laparoscopic insufflation of the abdomen depresses cardiopulmonary function. Surg Endosc, 1993; 7:12-16.

36. Feig BW, Berger BH, Dupuis JF et al - Hemodynamic effects of $\mathrm{CO}_{2}$ abdominal insufflation (CAI) during laparoscopy in high-risk patients. Anesth Analg, 1994;78:S-109.

37. Dhoste K - Haemodynamic and ventilatory changes during laparoscopic cholecystectomy in elderly ASA III patients. Can J Anaesth, 1996;43:783-788.

38. Haxby EJ, Gray MR, Rodriguez C - Assessment of cardiovascular changes during laparoscopic hernia repair using oesophageal Doppler. Br J Anaesth, 1997;78:515-519.

39. Hein HA, Joshi GP, Ramsay MA et al - Hemodynamic changes during laparoscopic cholecystectomy in patients with severe cardiac disease. J Clin Anesth, 1997;9:261-265.

40. Walder AD, Aitkenhead AR - Role of vasopressin in the haemodynamic response to laparoscopic cholecystectomy. $\mathrm{Br} \mathrm{J}$ Anaesth, 1997;78:264-266.

41. Zollinger A, Krayer S, Singer T et al - Haemodynamic effects of pneumoperitoneum in elderly patients with an increased cardiac risk. Eur J Anaesthesiol, 1997;14:266-275.

42. Breton G, Poulin E, Fortin C et al - Evaluation clinique et hémodynamique des cholécystectomies par voie laparoscopique. Ann Chir, 1992;45:783-790.

43. Liu SY, Leighton T, Davis I et al - Prospective analysis of cardiopulmonary responses to laparoscopic cholecystectomy. J Laparoendosc Surg, 1991;1:241-246.

44. Reid CW, Martineau RJ, Hull KA et al - Haemodynamic consequences of abdominal insufflation with $\mathrm{CO}_{2}$ laparoscopic cholecystectomy. Can J Anaesth, 1992;39:A132.

45. McLaughlin JG, Bonnell BW, Scheeres DE et al - The adverse hemodynamic effects related to laparoscopic cholecystectomy. Anesthesiology; 1992;77:A70. 
46. Westerband A, Van De Water J, Amzallag M et al - Cardiovascular changes during laparoscopic cholecystectomy. Surg Gynecol Obstet, 1992;175:535-538.

47. Cunningham AJ, Turner J, Rosenbaum S et al - Transoesophageal echocardiographic assessment of haemodynamic function during laparoscopic cholecystectomy. $\mathrm{Br} \mathrm{J}$ Anaesth, 1993;70:621-625.

48. Feig BW, Berger DH, Dougherty TB et al - Pharmacologic intervention can reestablish baseline hemodynamic parameters during laparoscopy. Surgery, 1994a;116:733-739.

49. Myre K, Rostrup M, Buanes T et al - Plasma catecholamines and haemodynamic changes during pneumoperitoneum. Acta Anaesthesiol Scand, 1998;42:343-347.

50. Viinamki O, Punnonen R - Vasopressin release during laparoscopy: role of increased intra-abdominal pressure. Lancet, 1982;16:175-176

51. Melville RJ, Frizis HI, Forsling ML et al - The stimulus for vasopressin release during laparoscopy. Surg Gynecol Obstet, 1985;161:253-256.

52. Joris JL, Chiche JD, Canivet JL et al - Hemodynamic changes induced by laparoscopy and their endocrine correlates: effects of clonidine. J Am Coll Cardiol, 1998;32:1389-1396.

53. Mann C, Boccara G, Pouzeratte Y et al - The relationship among carbon dioxide pneumoperitoneum, vasopressin release, and hemodynamic changes. Anesth Analg, 1999;89:278-283.

54. Koivusalo AM, Kellokumpu I, Scheinin M et al - Randomized comparison of the neuroendocrine response to laparoscopic cholecystectomy using either conventional or abdominal wall lift techniques. Br J Surg, 1996;83:1532-1536.

55. O'Leary E, Hubbard K, Tormey W et al - Laparoscopic cholecystectomy: haemodynamic and neuroendocrine response after pneumoperitoneum and changes in position. $\mathrm{Br} \mathrm{J}$ Anaesth, 1996;76:640-644.

56. Koivusalo AM, Scheinin M, Tikkanen I et al - Effects of esmolol on haemodynamic response to $\mathrm{CO}_{2}$ pneumoperitoneum for laparoscopic surgery. Acta Anaesthesiol Scand, 1998;42:510-517.

57. Ho HS, Gunther RA, Wolfe BM - Intraperitoneal carbon dioxide insufflation and cardiopulmonary functions. Laparoscopic cholecystectomy in pigs. Arch Surg, 1992;127:928-933.

58. Odeberg S, Ljungqvist O, Svenberg T et al - Haemodynamic effects of pneumoperitoneum and the influence of posture during anaesthesia for laparoscopic surgery. Acta Anaesthesiol Scand, 1994;38:276-283.

59. Hirvonen EA, Nuutinen LS, Vuolteenaho O - Hormonal responses and cardiac filling pressures in head-up or head-down position and pneumoperitoneum in patients undergoing operative laparoscopy. Br J Anaesth, 1997;78:128-133.

60. Iwase K, Takenaka H, Yagura A et al - Hemodynamic changes during laparoscopic cholecystectomy in patients with heart disease. Endoscopy, 1992;24:771-773.

61. Fox LG, Hein HAT, Gawey BJ et al - Physiologic alterations during laparoscopic cholecystectomy in ASA III \& IV patients. Anesthesiology, 1993;79:A55.

62. Smith I, Benzie RJ, Gordon NL et al - Cardiovascular effects of peritoneal insufflation of carbon dioxide for laparoscopy. Br Med J, 1971;14:410-411.

63. Goodale RL, Beebe DS, McNevin MP et al - Hemodynamic, respiratory, and metabolic effects of laparoscopic cholecystectomy. Am J Surg, 1993;166:533-537.

64. Ishizaki $Y$, Bandai $Y$, Shimomura K et al - Safe intraabdominal pressure of carbon dioxide pneumoperitoneum during laparoscopic surgery. Surgery, 1993;114:549-554.
65. Joris J, Honore P, Lamy M - Changes in oxygen transport and ventilation during laparoscopic cholecystectomy. Anesthesiology, 1992;77:A149.

66. Kazama T, Ikeda K, Kato $\mathrm{T}$ et al - Carbon dioxide output in laparoscopic cholecystectomy. Br J Anaesth, 1996;76:530-535.

67. Nunn JF, Hill DW - Respiratory dead space and arterial to end-tidal carbon dioxide tension difference in anesthetized man. J Appl Physiol, 1960;15:383-389.

68. Askrog $\mathrm{V}$ - Changes in (a-A) $\mathrm{CO}_{2}$ difference and pulmonary artery pressure in anesthetized man. J Appl Physiol, 1966;21: 1299-1305.

69. Bongard FS, Pianim NA, Leighton TA et al - Helium insufflation for laparoscopic operation. Surg Gynecol Obstet, 1993;177: 140-146.

70. Odeberg S, Sollevi A - Pneumoperitoneum for laparoscopic surgery does not increase venous admixture. Eur J Anaesthesiol, 1995; 12:541-548.

\section{RESUMEN}

Carraretto AR, Vianna PTG, Almeida AV, Ganen EM - Estudio Comparativo de los Efectos Hemodinámicos y Ventilatorios de la Ventilación Controlada a Volumen o a Presión, en Perros Sometidos a Pneumoperitoneo

JUSTIFICATIVA Y OBJETIVOS: La ventilación con presión controlada (PCV) está disponible en aparatos de anestesia, pero no existen estudios sobre su uso, durante el pneumoperitoneo con el $\mathrm{CO}_{2}$ (PPC). La finalidad de este estudio ha sido evaluar la ventilación controlada a presión, también como las alteraciones hemodinámicas y ventilatorias durante el PPC, comparándola con la ventilación controlada a volumen (VCV) convencionalmente utilizada.

MÉTODO: Dieciséis perros anestesiados con tiopental sódico, citrato de fentanil y bromuro de pancuronio, fueron divididos eventualmente en dos grupos: VC - ventilación controlada a volumen $(n=8)$ y PC - ventilación controlada a presión $(n=8)$ Los parámetros hemodinámicos y ventilatorios fueron monitorizados y registrados en 4 momentos: M1 (antes del $P P C$ ), M2 (30 minutos después del PPC = $10 \mathrm{mmHg}$ ), M3 (30 minutos después del $P P C=15 \mathrm{mmHg}$ ) y M4 (30 minutos después de la deflación del PPC).

RESULTADOS: Con la aplicación del PPC ocurrió un aumento del volumen corriente en el grupo PC, aumento de las presiones inspiratorias (máxima y de plato), disminución de la complacencia proporcional al aumento del PPC, aumento de la frecuencia cardiaca, mantenimiento de la presión arterial media con valores mayores en el grupo VC en todos los momentos, aumento de la presión del atrio derecho con disminución significativa después de la deflación, disminución del pH sanguíneo durante el PPC con menor variación en el grupo PC, mayor estabilidad de la presión parcial del $\mathrm{CO}_{2}$ en la sangre arterial en el grupo $P C$, sin alteraciones de la presión parcial de la $\mathrm{O}_{2}$ en la sangre arterial.

CONCLUSIONES: A pesar de las diferencias de algunos parámetros hemodinámicos y ventilatorios, entre los dos modos de control de la ventilación, en las condiciones estudiadas fue posible la utilización de la ventilación controlada a presión para procedimientos con la aplicación del $P P C$. Es fundamental observar el control riguroso de la ventilación alveolar, ajustando la presión inspiratoria para mantener una eliminación adecuada del $\mathrm{CO}_{2}$ y garantizar oxigenación. 